\title{
Statistical Characteristics and Community Analysis of Urban Road Networks
}

\author{
Wen-Long Shang $\mathbb{D},{ }^{1}$ Yanyan Chen, ${ }^{1}$ Huibo Bi $\mathbb{D}^{1},{ }^{1}$ Haoran Zhang, ${ }^{2}$ Changxi Ma $\mathbb{D},{ }^{3}$ \\ and Washington Y. Ochieng ${ }^{4}$ \\ ${ }^{1}$ Beijing Key Laboratory of Traffic Engineering, College of Metropolitan Transportation, Beijing University of Technology, \\ Beijing 100124, China \\ ${ }^{2}$ Centre for Spatial Information Science, The University of Tokyo, Tokyo 277-8563, Japan \\ ${ }^{3}$ School of Traffic and Transportation, Lanzhou Jiaotong University, Lanzhou 730070, China \\ ${ }^{4}$ Centre for Transport Studies, Imperial College London, London SW7 2AZ, UK
}

Correspondence should be addressed to Huibo Bi; huibobi@bjut.edu.cn

Received 13 March 2020; Revised 9 August 2020; Accepted 25 August 2020; Published 9 September 2020

Academic Editor: Jos Manuel Galn

Copyright ( $) 2020$ Wen-Long Shang et al. This is an open access article distributed under the Creative Commons Attribution License, which permits unrestricted use, distribution, and reproduction in any medium, provided the original work is properly cited.

\begin{abstract}
Urban road networks are typical complex systems, which are crucial to our society and economy. In this study, topological characteristics of a number of urban road networks purely based on physical roads rather than routes of vehicles or buses are investigated in order to discover underlying unique structural features, particularly compared to other types of transport networks. Based on these topological indices, correlations between topological indices and small-worldness of urban road networks are also explored. The finding shows that there is no significant small-worldness for urban road networks, which is apparently different from other transport networks. Following this, community detection of urban road networks is conducted. The results reveal that communities and hierarchy of urban road networks tend to follow a general nature rule.
\end{abstract}

\section{Introduction}

Urban road networks (URNs) are vital in underpinning our society and economy [1]. Particularly, nowadays with the growing popularity of technologies related to smart city and cooperative vehicle infrastructure systems (CVIS), physical roads and their constituent URNs actually carrying most of urban traffics are increasingly receiving attention. Meanwhile, URNs can be described as typical complex networked systems [2] as there are large numbers of spatial components with complex structures and interactions between different components. Since Watts and Strogats [3] and Barabasi and Albert [4] proposed "small-world" networks and scale-free networks, respectively, complex network theory has been considered as a good tool to observe and analyse topological and structural features of networked systems [5].

In fact, complex network theory has been applied to the study of various transport networks, such as public transport
[6-9], air transport $[10,11]$, railway transport $[12,13]$, and marine transport $[14,15]$. Sienkiewicz and Hołyst [16] explored the public transport network (PTN) systems of 22 cities in Poland, and the results show that topological characteristics of these cities, such as degree and degree distribution, average path length, and cluster coefficients, tend to follow power-law distribution and exponential distribution, and these PTNs also demonstrate significant small-worldness. Following this, Xu et al. [17] investigated the topological properties of three bus-transport networks in Beijing, Shanghai, and Nanjing. The degree distribution in the three cities shows power laws in space $L$ (nodes refer to stations, and links denote that at least one route exists between two consecutive stations), while in space $P$ (nodes denote stations, while links exist when two stations are shared by at least one route), the cumulative degree distribution follows an exponential distribution. Similarly, Von Ferber et al. [6] explored the public transport networks 
(PTNs) in 14 cities, and the results show that these PTNs have high clustering coefficients and relatively low average shortest paths, which are typical small-world characteristics. A directed and weighted bus-transport network in Beijing also suggests that this bus network is a small-world network [18]. In order to examine the relationship between city size and network centrality, Derrible [19] investigated betweenness centrality of 28 metro systems across the world. Dimitrov and Ceder [7] examined the structure and topological characteristics of the bus network in Auckland, New Zealand, based on the bus routes. As well as this, Sun et al. [20] analysed topological characteristics of the Beijing rail transit network (BRTN), and vulnerable stations and cascading failures of the BRTN were studied under different intentional attacks based on degree, betweenness, and strength indices. De Regt et al. [21] analysed the topological and spatial characteristics of PTNs in the UK, and PTNs such as London, Manchester, West Midlands, and national rail were investigated to discriminate their stability. Particularly, they found the universal load dynamics of these systems, which is useful for the efficiency of the PTN.

In addition to public transport networks, statistical characteristics of air networks $[10,11,22]$, railway networks $[12,13]$, and cargo ship networks $[14,15]$ have also been explored based on complex network theory. Although complex network theory has been frequently used to study many types of transport networks, complex network research related to urban road networks (URNs) consisting of road segments and junctions, which directly carry most of urban traffics, is very limited. Jiang et al. [23] attempted to characterize the topology structure of six urban street networks from the perspective of degree correlation, and they found that the reshuffled networks from six street networks tend to deviate the small-world properties compared to the original networks. Following this, Kirkley et al. [24] mainly focused on the betweenness centrality of 97 cities worldwide to demonstrate the invariance of its statistical distribution for planar networks. In addition, other previous studies either investigated certain city road networks in dual representation (nodes refer to the set of edges sharing common attributes) so as to observe the growth of the city or model the congestion $[25,26]$ or focused on the robustness and response of road networks under different attack strategies [27, 28]. However, several questions related to URNs are still unclear: for instance, compared with other transport networks, what are the common statistical characteristics of URNs in different countries? How are the small-worldness and underlying relationships between the statistical characteristics? How are the community and hierarchy features of URNs and what kind of rules they may follow? This study is conducted and organized based on these questions, and answering these questions is the central message that we are trying to deliver. To answer these questions, this paper investigates the topological characteristics of urban road networks from a number of cities in Europe and North America in order to explore substantial structure features of these networks. In addition, small-world analysis and community detection are also conducted.
The rest of this paper is organized as follows. Section 2 introduces the widely used topological indices in the field of network science. Section 3 presents the statistical analysis of the urban road networks, and the small-world analysis is also conducted. Following this, correlation analysis between some topological indices is investigated in Section 4. Section 5 explores community detections of the urban road networks, and finally, we draw conclusions in Section 6. The structure of our study is logically organized, and previous sections can support the subsequent ones. For example, Sections 4 and 5 are both based on statistical analysis of topological indices of the URNs.

\section{Background and Database}

In this paper, complex network theory is employed to explore topological characteristics of urban road networks (URNs) as it is a good tool to study very complex systems. Data associated with the URNs used in this study are obtained from a website (http://www.bgu.ac.il/ bargera/tntp/), which is frequently used for transportation problems. Unlike typical space $L$ and space $P$ representations [29] and dual information representation [30], the nodes of an urban road network refer to the intersections of urban streets or roads, while the links represent the street or road segments connecting nodes. It is worth noting that the construction of URNs is not our focus, and we directly use the URN data from the website (http://www.bgu.ac.il/ bargera/tntp/) developed by the scholars in the field of transportation. Due to the lack of traffic data, we do not take into account traffic on the roads. In addition, all URNs used in the study are connected. Urban road networks can be divided into weighted or unweighted based on whether links are labelled with a weight, which can be the length of links or traffic flow on such links, and so on [31]. The weight of links depends on the purpose of research.

The URNs used in this paper mainly represent cities in Europe and North America because of the limited scope of data sources. Specifically, these URNs are Austin, Chicago, Philadelphia, Anaheim, Winnipeg, Central Berlin, Barcelona, Terrassa, and Hessen, and the size of the URNs varies, ranging from 416 nodes to 13,389 nodes. Such information including the number of links is presented in Table 1.

Table 2 summarises six topological indices used in this paper: degree, clustering coefficient, average path length (APL), closeness centrality, betweenness centrality, and efficiency. In order to distinguish global and local indices [34], efficiency and APL are presented at the last two rows, and these two indices may present the global performance of URNs from different perspectives. Here, we use these six indices to analyse topological properties of URNs.

\section{Statistical Analysis of the Urban Road Networks}

Due to the fact that the complexity of urban road networks is partially arising from the topological characteristics and underlying structure features, statistical analysis plays a vital 
TABLE 1: Urban road networks with different scales.

\begin{tabular}{lccc}
\hline City & Nodes & Links & Locations \\
\hline Austin & 7388 & 18,961 & America \\
Chicago & 12,982 & 39,018 & America \\
Philadelphia & 13,389 & 40,003 & America \\
Anaheim & 416 & 914 & America \\
Winnipeg & 1052 & 2836 & America \\
Berlin & 12,981 & 28,376 & Europe \\
Barcelona & 1020 & 2522 & Europe \\
Terrassa & 1609 & 3264 & Europe \\
Hessen & 4660 & 6674 & Europe \\
\hline
\end{tabular}

TABLE 2: Six indices used for analysing urban road networks.

\begin{tabular}{|c|c|c|}
\hline Indices & Equations & Interpretation \\
\hline Degree & $k_{i}=\sum_{j} \alpha_{i j}$ & $\begin{array}{l}\alpha_{i j} \text { is the connection between node } i \text { and node } j \text {; if } i \text { and } j \text { are connected, } a_{i j} \text { is } 1 \text {; } \\
\text { otherwise, it is } 0 \text {. The mean degree of a network, denoted as } k \text {, is the average of the } \\
\text { degree of all its nodes [32]. The degree distribution of the network } P(k) \text { is a ratio of } \\
\text { the number of nodes with degree } k \text { to the number of all nodes and represents the } \\
\text { cumulative degree distribution. }\end{array}$ \\
\hline $\begin{array}{l}\text { Clustering } \\
\text { coefficient }\end{array}$ & $C c(i)=\left(A_{i} /\left(\left(k_{i}\left(k_{i}-1\right)\right) / 2\right)\right)$ & $\begin{array}{l}A_{i} \text { is the actual number of edges among the neighbours of node } i \text { (nodes connected } \\
\text { with node } i \text { are called its neighbours); } k_{i} \text { is the number of the neighbours of node } i \text {, } \\
\text { namely degree of node } i[3] .\end{array}$ \\
\hline $\begin{array}{l}\text { Closeness } \\
\text { centrality }\end{array}$ & $C_{i}=\left((n-1) / \sum_{v_{j} \in V, i \neq j} d_{i j}\right)$ & $\begin{array}{l}V \text { is the set of nodes, } V=\left\{v_{j}: j=1,2, \ldots, n\right\} \text {, and } n \text { is the number of nodes. } d_{i j} \text { is } \\
\text { the length of the shortest path between } i \text { and } j \text {. For unweighted networks (i.e., every } \\
\text { link has an equal length of } 1), d_{i j} \text { is the number of links of the shortest path } \\
\text { between } i \text { and } j \text {, while for weighted networks, assuming that the weight is simply } \\
\text { physical distance, } d_{i j} \text { is the physical distance of the shortest path from node } i \text { to } j \text {. }\end{array}$ \\
\hline $\begin{array}{l}\text { Betweenness } \\
\text { centrality }\end{array}$ & $B_{i}=\sum_{z \neq j \neq i \in V}\left(\sigma_{z j}(i) / \sigma_{z j}\right)$ & $\begin{array}{l}\sigma_{z j} \text { is the total number of shortest paths between nodes } v_{z} \text { and } v_{j} \text {, and } \sigma_{z j}(i) \text { is the } \\
\text { number of shortest paths passing through node } i \text {. Weighted betweenness centrality } \\
\text { (WBC) takes the actual distance of the link as the edge weight. }\end{array}$ \\
\hline $\begin{array}{l}\text { Average path } \\
\text { length }\end{array}$ & $L=(1 / n(n-1)) \sum_{i>j} d_{i j}$ & $\begin{array}{l}d_{i j} \text { is the length of the shortest path between } i \text { and } j . \text { For unweighted networks (i.e., } \\
\text { every link has an equal length of } 1), d_{i j} \text { is the number of links of the shortest path } \\
\text { between } i \text { and } j .\end{array}$ \\
\hline Efficiency & $E(G)=(1 / n(n-1)) \sum_{i \neq j \in G}\left(1 / d_{i j}\right)$ & $\begin{array}{l}\text { This index was first proposed by Crucitti et al. [33] to explore the global efficiency } \\
\text { of complex networks and looked very similar to APL; in this study, actual distance } \\
\text { is assigned to the weight of a link. The greater } E \text {, the better the efficiency. }\end{array}$ \\
\hline
\end{tabular}

role in understanding such complexity and explores substantial mechanisms of urban road networks.

In this study, in order to capture more realistic topological characteristics of URNs, we regard these URNs as directed networks, and roads or streets only for pedestrians are not taken into account. According to the background provided in Section 2, topological indices of these URNs are calculated in order to explore topological characteristics and discover the underlying properties of the urban road networks (URNs) under consideration.

3.1. Degree Centrality. Degree is a seemingly simple yet very important index since it reflects the node's connectivity and its importance in its vicinity. Therefore, this index is used to measure the local centrality of the networks. The degree centrality $k$ of a node $i$ in an undirected network (i.e., the links are nondirectional) is the number of links connecting to this node [4]. In realistic URNs, each intersection of streets/roads is regarded as a node, while the link represents the street/road segments between the nodes. Here, nine URNs used in this study are directional; therefore, the degree is divided into in-degree (the number of links leading to a given node) and out-degree (the number of links leading away from a given node). The average in-degree and outdegree of nine URNs have been summarised in Table 3, and the degree distributions of nine URNs in the log-linear scale are illustrated in Figure 1.

In Figure 1, the $y$-axis represents logarithm of the complementary cumulative probability $P(K \geq k)$, which is the probability that a randomly chosen URN node has indegree/out-degree which equals to or larger than $k$. Given that the degree denotes the number of streets connecting with junctions of roads, it is expected that the range of indegree and out-degree for these URNs is not too large. For example, although the Philadelphia road network has 13,389 nodes and 40,003 links, the maximum in-degree and outdegree are both 4. Among the nine URNs, Barcelona and Hessen have, respectively, the largest and second-largest ranges of degree, despite their smaller sizes. The slope of the distribution curves in Figure 1 describes the speed of descent of the in-degree and out-degree distribution curves, which reflects whether the majority of nodes of URNs is less connected. For example, Hessen shows the steepest slope of 
TABLE 3: Indices of nine urban road networks.

\begin{tabular}{|c|c|c|c|c|c|c|c|c|}
\hline City & $\begin{array}{c}\text { Average in- } \\
\text { degree }\end{array}$ & $\begin{array}{l}\text { Average out- } \\
\text { degree }\end{array}$ & $\begin{array}{c}\text { Average } \\
\text { betweenness }\end{array}$ & Closeness & $\begin{array}{l}\text { Clustering } \\
\text { coefficient }\end{array}$ & APL & Diameter & Efficiency \\
\hline Austin & 2.5658 & 2.5658 & 0.0099 & 0.0393 & 0.0010 & 48.58 & 118 & 0.0284 \\
\hline Chicago & 3.0062 & 3.0062 & 0.0053 & 0.0273 & 0.0017 & 44.71 & 111 & 0.0297 \\
\hline Philadelphia & 2.9878 & 2.9878 & 0.0059 & 0.0446 & 0.0007 & 43.17 & 98 & 0.0290 \\
\hline Anaheim & 2.1971 & 2.1971 & 0.0316 & 0.00003 & 0.0157 & 11.87 & 31 & 0.1098 \\
\hline Winnipeg & 2.7269 & 2.7269 & 0.0253 & 0.0897 & 0.0007 & 18.85 & 40 & 0.069 \\
\hline Berlin & 2.1855 & 2.1855 & 0.0075 & 0.0001 & 0.0017 & 50.09 & 116 & 0.0242 \\
\hline Barcelona & 2.7118 & 2.7118 & 0.0203 & 0.1853 & 0.0009 & 14.00 & 31 & 0.0765 \\
\hline Terrassa & 2.0362 & 2.0362 & 0.0181 & 0.3441 & 0.0009 & 25.09 & 64 & 0.0533 \\
\hline Hessen & 1.4322 & 1.4322 & 0.0160 & 0.0516 & 0.0003 & 45.60 & 137 & 0.0282 \\
\hline
\end{tabular}

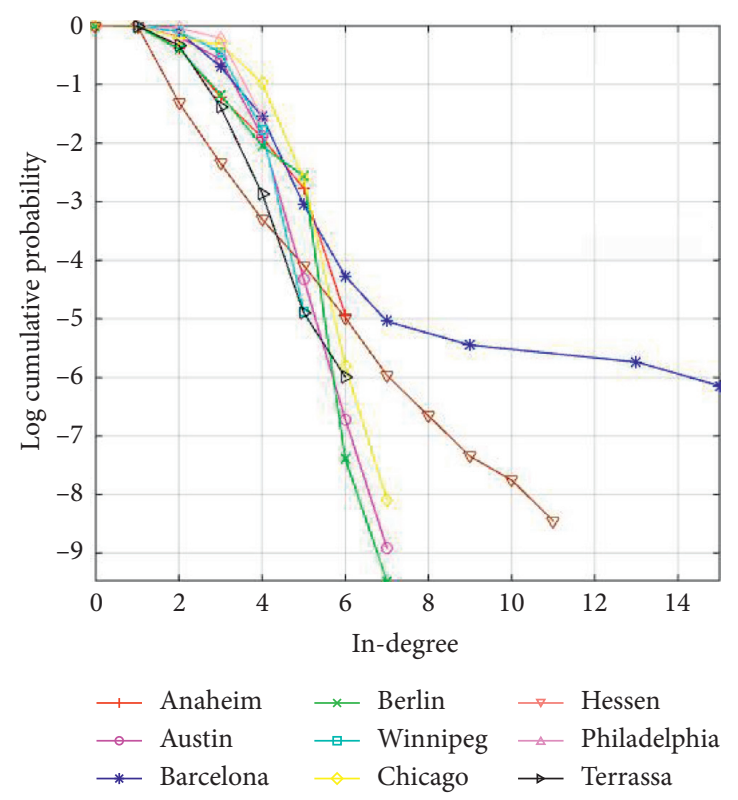

(a)

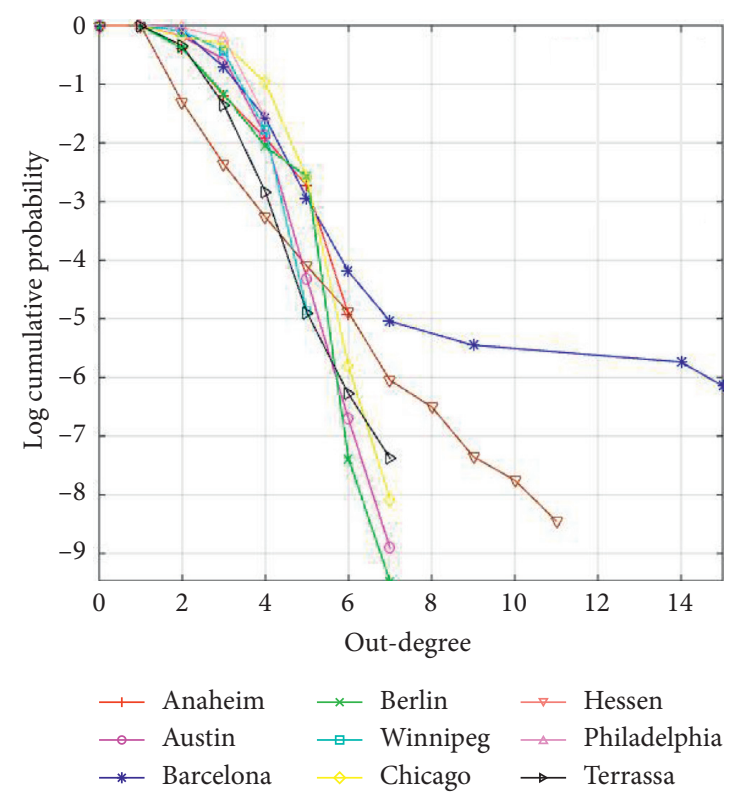

(b)

FIGURE 1: In-degree and out-degree distributions of urban road networks in the log-linear scale.

the distribution curve, which suggests that compared to other URNs, the proportion of nodes of Hessen with low indegree/out-degree is the largest, so overall, Hessen is less connected than other URNs. In this study, the statistical significance of degree distribution is not very significant, given that the range of in- and out-degree is limited. Here, the degree distribution is mainly used to present the proportion of each value of in- and out-degree so as to explore the structural properties of the URNs.

Unlike the rest of the URNs, the in-degree/out-degree of Barcelona has significant portions that are larger than 9, and the curves for Barcelona consist of two segments: the first one decreasing quickly, while the second decreases slowly. The explanation is that the proportion of nodes with higher in- and out-degree (larger than 9) for Barcelona is relatively higher than other URNs, which implies that the nodes of the Barcelona network are more likely to connect with more roads compared to other URNs. However, given the lack of coordinate data and geographical information, it is difficult for us to know how exactly the junctions in Barcelona connect with other roads by visualization. Figure 1 shows that most of nodes in these nine URNs are connected through a few nodes and that the distributions of in-degree and out-degree are qualitatively similar, which suggest that most of street/road segments in these nine URNs are bidirectional. We also plot the degree distributions of the URNs in the log-log scale in Figure 2, and it shows that the degree distribution is very similar with that in Figure 1.

The second and the third columns in Table 4 show that most of the in-degree and out-degree distributions follow the power law well. This result is consistent with the previous work relating to public transport networks [6, 16, 35], worldwide maritime transportation networks represented in space $L$ [14], and the Chinese bus-transport network [17]. It is worth noting that the in-degree/out-degree distributions of some URNs also follow other distributions very well, for example, Anaheim, Berlin, and Austin can also obey Gaussian distribution: $P(k \geq x) \propto e^{-(x-b 1 / c 1)^{2}}$, and the goodness for in-degree is $0.98,0.95$, and 0.96 , respectively, even so it is still enough to illustrate the decaying tendency of in-degree/out-degree. In addition, the distributions of indegree and out-degree for these URNs show similarity, and 


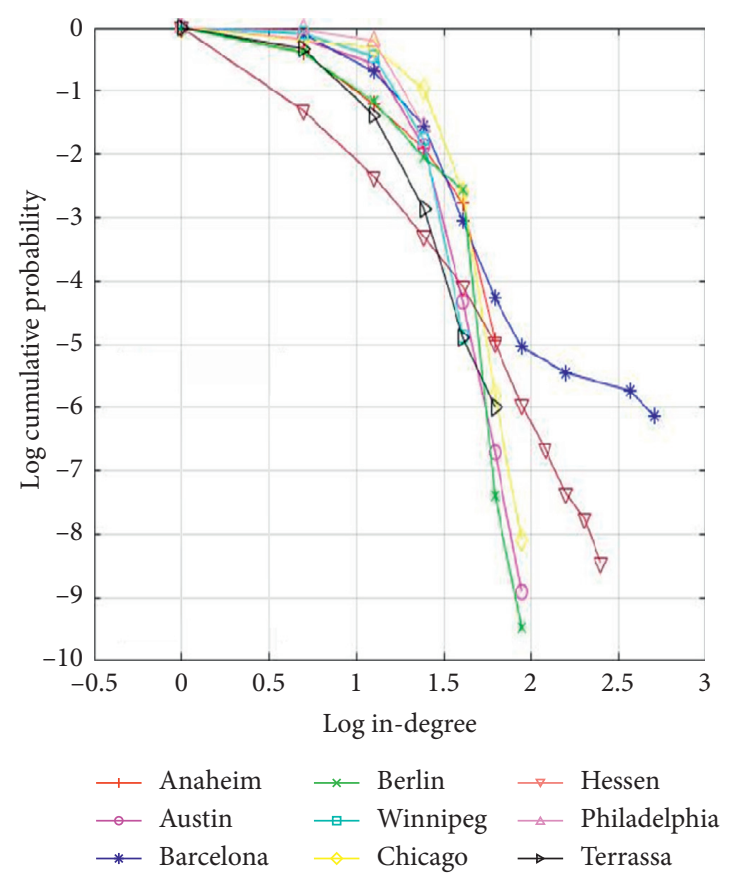

(a)

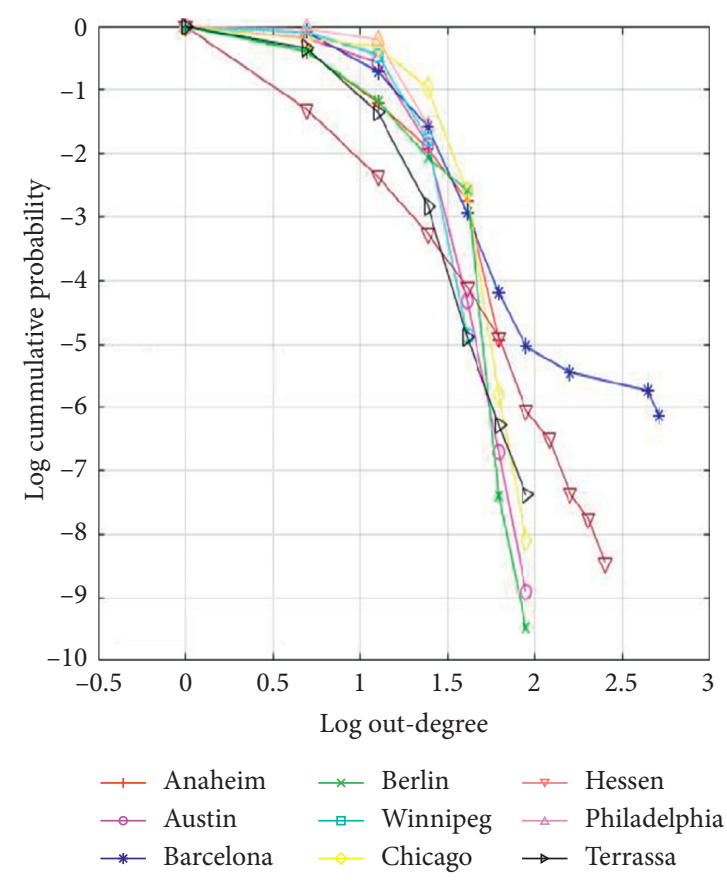

(b)

Figure 2: In-degree and out-degree distributions of urban road networks in the log-log scale.

this confirms that most of the links within the nine URNs are once again bidirectional. All node degree distributions are shown to follow the power law well, $P(k \geq x) \propto x^{-\mu}$, except that Barcelona follows a two-regime power law, and $\mu$ is the slope of the fitted curve (in the logarithmic coordinates), which depicts the speed of descent of the in-degree and outdegree distribution curves. A distribution curve with a steep slope suggests that the majority of nodes has smaller values of in-degree/out-degree given that it is in the logarithmic coordinates; in other words, the URN overall is less well connected. Philadelphia, with the steepest slope among the nine URNs, is overall less well connected than the other URNs.

3.2. Clustering Coefficient. The clustering coefficient $(C C)$ is used to measure the local compactness of a network and presents the clustering effect and local features of the network. A larger $C C$ of a given node means the neighbours of the node are more likely to attract each other so that the local area of the network is more compact. According to the definition of CC in Table 2, the CC for all URNs is calculated. Figure 3 presents the logarithmic complementary cumulative distribution, $P(C c \geq x)$, of $C C$.

As can be seen from Figure 3, the distribution for Hessen has the steepest absolute slope, which means the road network of Hessen is the loosest compared to other eight cities due to the fact that most of its nodes have smaller values of $C C$ followed by Winnipeg and Philadelphia. The URN of Terrassa is looser than Barcelona, Chicago, Berlin, and Austin, and the nodes of Anaheim tend to attract each other within the local range more than the other urban road networks in this study. The average CC for the nine URNs has been summarised in Table 3, which is normalized by the number of nodes and also proves the viewpoint observed from Figure 3. The average CC of these URNs is smaller compared to the Chinese airport network [36], the Italian airport network [11], the worldwide air transportation network [10], and the Boston subway network [37], possibly because the neighbouring nodes of a given node in the URNs are less likely to be connected to each other. This index is also able to be used to judge whether the network has smallworld properties [3], and it will be depicted in detail later.

3.3. Weighted Closeness Centrality (WCC). As previously introduced in Table 2, closeness centrality [38] of node $i$ is the inverse of the sum of the shortest path distance from node $i$ to other nodes, and it measures the accessibility of networks. In this study, the actual distances of links are taken as the link weights. According to the function in Table 2, the WCC for nine URNs is calculated. The cumulative distributions of WCC for these nine URNs are presented in Figure 4 in a logarithmic scale. Given the differences in the size of these URNs, weighted closeness centrality is also normalized.

As can be seen from Figure 4, all nodes of Anaheim and Berlin have relatively smaller WCC, which demonstrates that these two URNs are less accessible from a given node to other nodes, while Terrassa, Barcelona, and Winnipeg have relatively large $W C C$. This conclusion is consistent with the result based on the average weighted closeness shown in Table 3.

The WCC distribution is fitted for the nine URNs, as presented in Table 4. As observed from the fourth column in Table 3, most URNs follow an exponential distribution, 


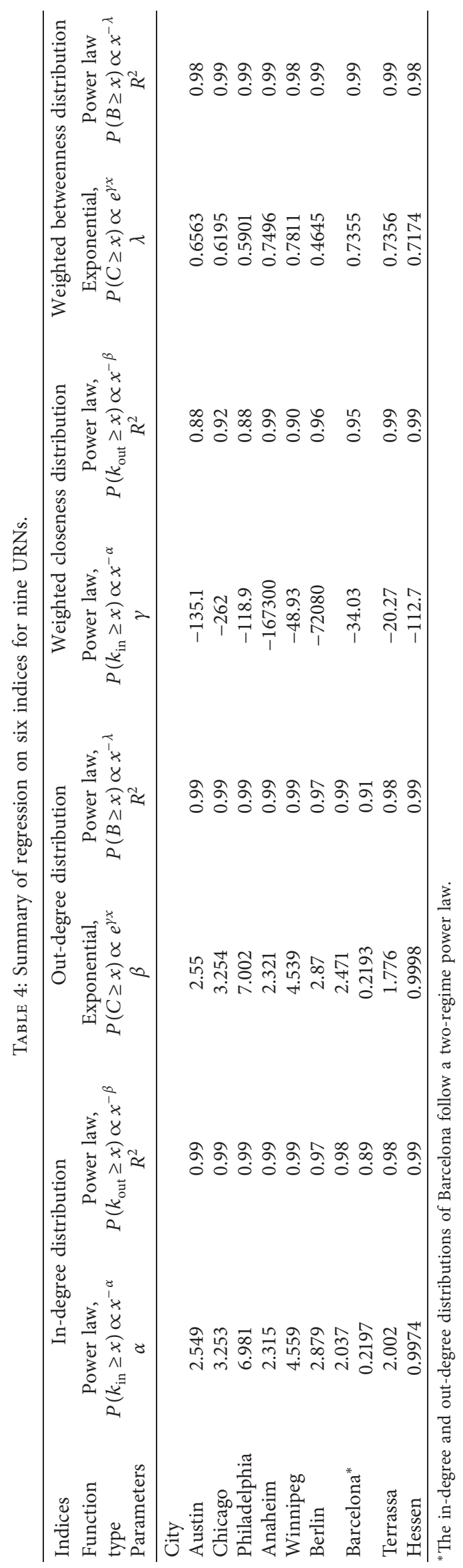




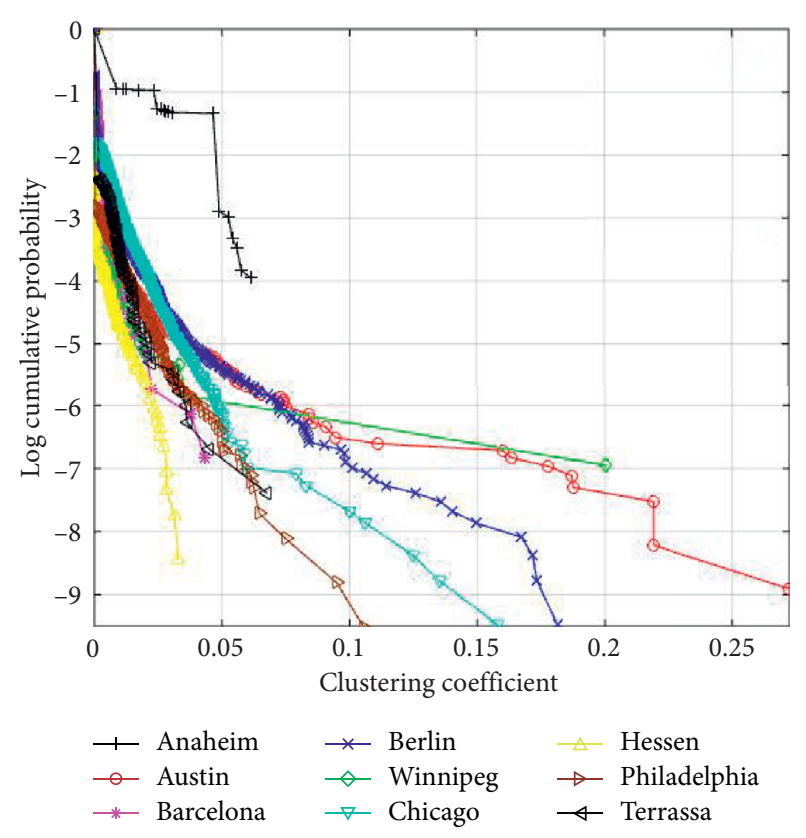

FIGURE 3: Clustering coefficient of URNs.

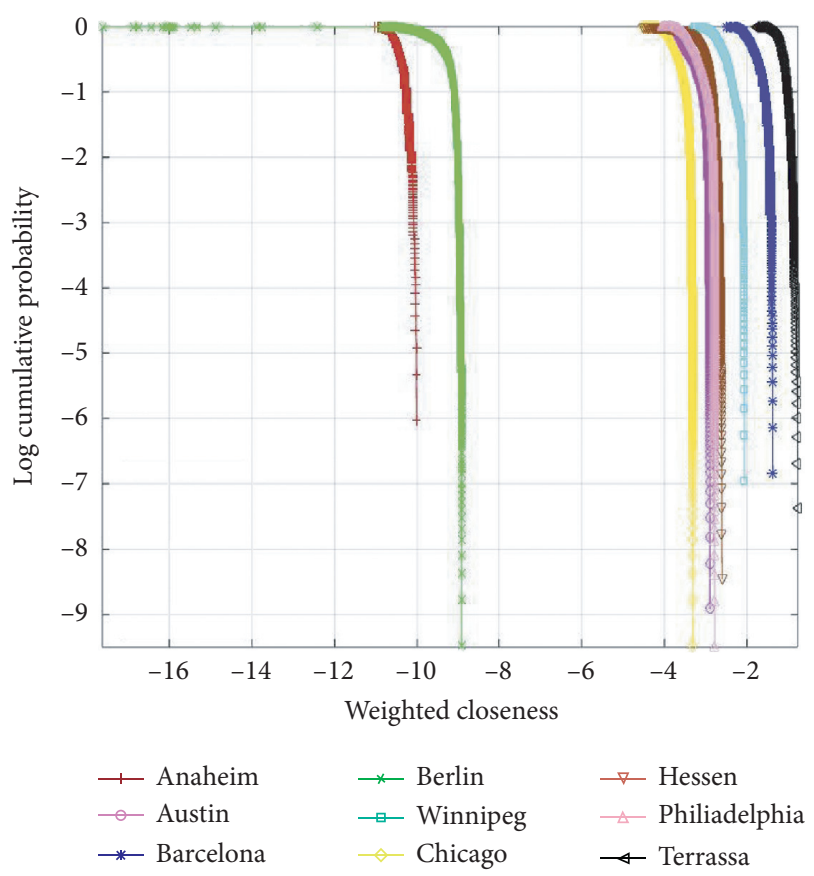

FIgURE 4: Weighted closeness centrality of URNs.

i.e., $P(C \geq x) \propto e^{\gamma x}$, yet Austin, Philadelphia, and Winnipeg do not fit this exponential distribution as well as other networks. From this distribution, it can be seen that Anaheim and Berlin have the largest $\gamma$ parameter values, which suggests that most of the nodes in these two URNs have smaller WCC followed by Chicago, Austin, Philadelphia, and Hessen, and Terrassa and Barcelona have a smaller $\alpha$-value. This is consistent with what Figure 4 describes.

The average closeness centrality for nine URNs is summarised in Table 3 . The nodes with the largest WCC are generally thought as being located in the geographic centre of the city since these nodes are easier to access than the other nodes.

3.4. Weighted Betweenness Centrality (WBC). Betweenness is a very important topological index in that it indicates that how frequently a given node is passed through by the shortest paths between all node pairs. In this study, the length of links is used as the edge weight, and $W B C$ is calculated according to the function in Table 2. The logarithmic complementary cumulative distribution of WBC for the nine URNs is presented in Figure 5. In order to mitigate the effect of the size of different networks to betweenness, the weighted betweenness is normalized by dividing $n(n-1)$, where $n$ is the node number of the network. Figure 5 exhibits that most of the nodes for the nine URNs have small $W B C$, and among all URNs, by intuition, Chicago overall shows the fastest decaying behaviour followed by Philadelphia and Austin, while Anaheim presents a relatively flat decaying tendency followed by Winnipeg and Barcelona. As discussed, the larger betweenness centrality of a given node means the node is more important due to the fact that more of the shortest paths in the URN pass through this node. Betweenness centrality can be related to the robustness. In the transportation field, the nodes with high BC tend to be regarded as very important ones since these nodes are most likely to be used by travellers or to be allocated crucial resources, and the travel time and congestion would increase greatly if such nodes are subject to malfunctions caused by disruptions. Therefore, these nodes are most vulnerable parts of the whole network system. According to Sun et al. [39], a network is less robust if the proportion of nodes with large betweenness centrality is higher. The slopes of the curves in Figure 5 show this proportion statistically; in other words, the steeper the slope is, the more robust the URN is.

The logarithmic complementary cumulative distribution of $W B C$ can also be fitted by a power-law function, $P(B \geq x) \propto x^{-\lambda}$, which is identical to Kirkley et al.'s finding [24], and the results for this are summarised in Table 4. In this case, normalized $B \in(0,1)$; thus, the larger $\lambda$, the slower the rate of decay.

Comparing all $\lambda$, Berlin exhibits the quickest decaying characteristic of the WBC distribution followed by Philadelphia, Chicago, and Austin, which is against the intuitive observation. The reason could be that the distributions tend to overestimate or underestimate the decaying tendency of the curves in this case. The average weighted betweenness centrality $(A W B C)$ of these URNs, however, which is presented in Table 3, shows that Chicago has the smallest value followed by Philadelphia, Berlin, and Austin and that Anaheim has the largest value followed by Winnipeg and Barcelona. Statistically, the lower the $A W B C$ the network has, the more robust the network is [39]. The conclusion related to the robustness of these networks based on $A W B C$ is therefore roughly consistent with that of observations from Figure 5.

$W B C$ depicts how a given node of the network is passed by the shortest paths, and this information may be used for the allocation of important resources. Hence, this index is 


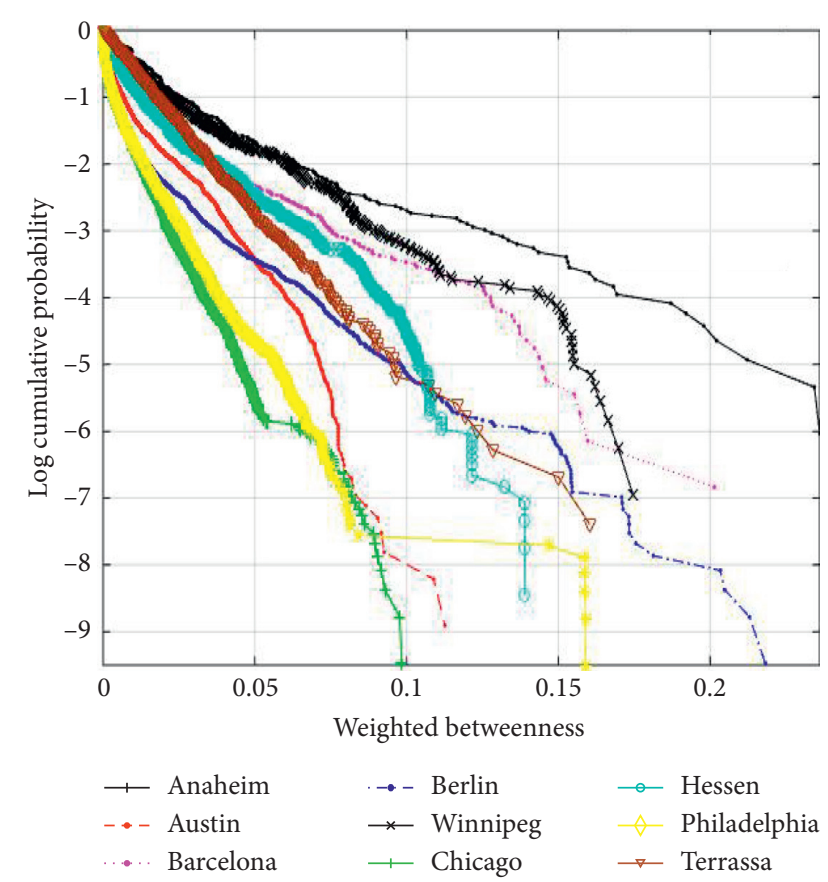

FIGURE 5: Weighted betweenness centrality of nine URNs.

sometimes used to assess the robustness and vulnerability of the network [39].

3.5. Average Path Length (APL), Diameter, and Efficiency. $A P L$, also called the characteristic path length, can be employed to quantify the structural characteristics of networks [3]. The diameter $(D)$ of the network is the maximum $d_{i j}$ for all node pairs. Here, $A P L$ can be categorized as weighted and unweighted. The weighted $A P L$ tends to measure the distance travelled from a given node to another node, while the unweighted $A P L$ assesses how many links need to be traversed from a given node to another. Both are able to assess the degree of accessibility of the URN. This study analyses unweighted $A P L$ to explore the structure of these URNs since there are no units for the distance data for the nine urban road networks, which means that comparisons of absolute values of APL between different URNs are meaningless. Here, we need to point out that, unlike $A P L$, the distribution analysis of $W B C$ and $W C C$ for the URNs is not impacted by the distance data without units since their calculations are based on the normalization for statistical distribution analysis. Furthermore, geodesic distance, namely, the number of links connecting a node pair, is more focused in this study because of the homogenous characteristics of the physical links of each individual network. Accordingly, the APL and the diameter for the nine URNs have been summarised in Table 3. As shown in Table 3, compared to other indices, we do not normalize $A P L$ and diameter and just present the absolute values of them so as to directly to show how many links can be passed averagely by shortest paths between any node pairs of the URNs and what the maximum shortest path for all node pairs is. Therefore, APL and diameter seemingly vary greatly among them and are larger than other indices. Anaheim has the smallest $A P L$, which means the nodes of this network can be more efficiently connected by paths. This is followed by Barcelona and Winnipeg, while the three networks with the largest APL are Austin, Hessen, and Berlin. The diameter and the $A P L$ of the nine URNs are strongly correlated with the coefficient 0.9714 according to a Pearson correlation. In some cases, $A P L$ and the diameter of networks are used to assess network performance $[33,40,41]$. The correlations between the size of networks, $A P L$, and diameter are shown in Figure 6.

As can be seen from Figure 6, the Pearson correlations between $A P L$, diameter, and the size of networks are 0.8358 and 0.7223 , respectively. This demonstrates that these two indices are positively proportional to the number of nodes to some extent. Here, we need to emphasize that it is expected that the larger city is more likely to have larger $A P L$ and diameter, and our correlation analysis has also proved this point. Accurately quantifying the correlation coefficients between $A P L$, diameter, and the size of the URNs is also very important for the topological analysis of URNs.

In this study, $A P L$ is calculated based on the function in Table 2. If a network suffers from severe destruction, which may be generated by internal or external disruptions so that the network is disconnected, an extremely large value is assigned to the disconnected links for the calculation of APL. However, this approach has no effect on the ranking of link importance, and comparing the absolute value of $A P L$ does not make sense. In order to better measure the performance of unconnected networks [33], an efficiency measure based on the inverse of the shortest path length is proposed in order to better observe the variations in the efficiency of different networks. According to the efficiency function presented in Table 2, the results of efficiency for the nine URNs are calculated and also summarised in Table 3. As can be seen, the results are consistent with the $A P L$; for example, Anaheim has the smallest $A P L$ (11.87), and, correspondently, its efficiency measure is the largest among all nine URNs, while Berlin has the largest APL (50.09) and the smallest efficiency. In general, efficiency is able to be used to measure the performance of networks when suffering from disturbances.

In this study, we mainly utilize commonly used topological indices to explore the statistical characteristics of selected nine URNs, which are located in America and Europe. As can be seen from Table 4, there seems to be some differences between American and European cities, that is, parameter $\alpha$ of the in-degree distribution for European cities is smaller than that for American cities, except for the Berlin network. However, due to the limited number of the URNs, we cannot confirm this point. Based on our intuition, some different characteristics of URNs in different countries/ continents probably exist owing to different history, culture, levels of economic development, and so on, but a large number of sample data are really needed to support. Here, we cannot conclude the differences between American and European cities due to limited data sources.

In order to further understand the topological characteristics of the URNs, we calculate the topological indices of 


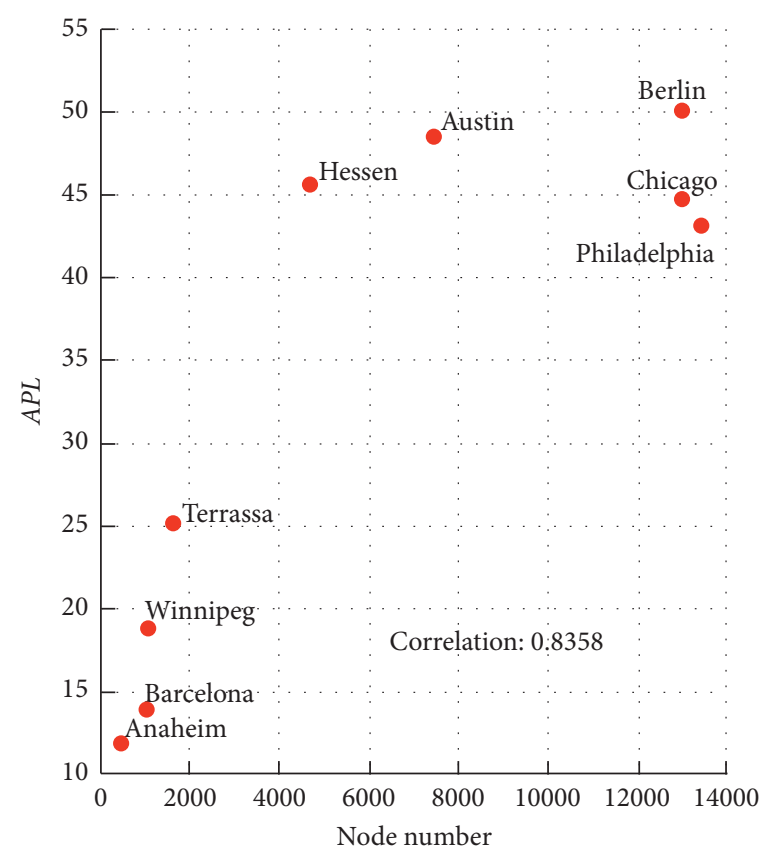

(a)

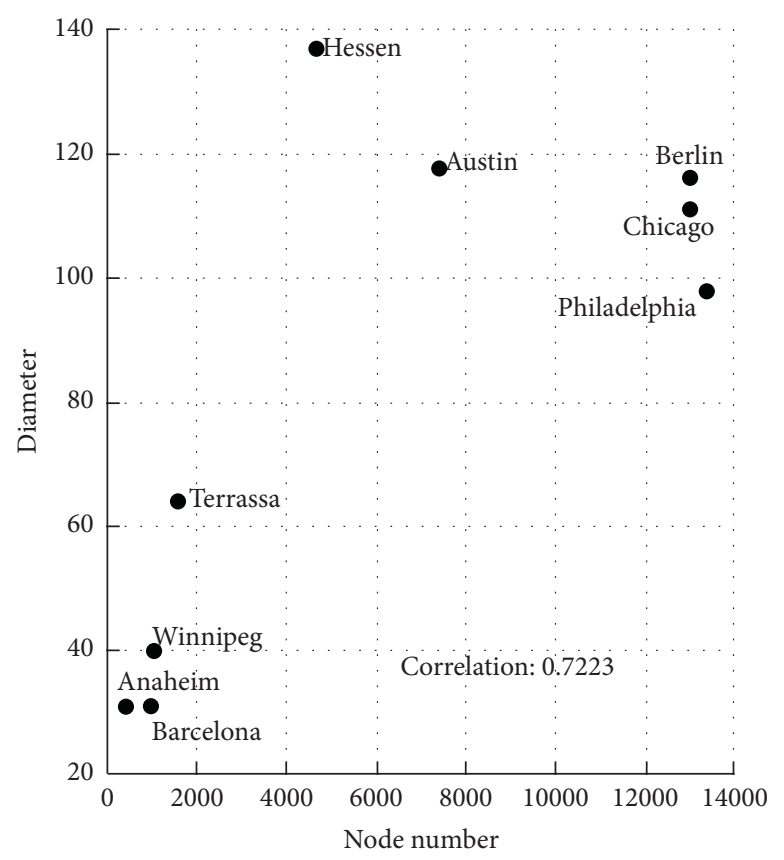

(b)

Figure 6: Correlations between APL, diameter, and the size of nine URNs.

TABLE 5: Indices of ER graphs corresponding to the URNs.

\begin{tabular}{|c|c|c|c|c|c|}
\hline City & Average degree & Average betweenness & Clustering coefficient & APL & Diameter \\
\hline ER_Austin & 4.9349 & 0.0103 & 0.0002 & 9.15 & 23 \\
\hline ER_Chicago & 5.9954 & 0.0039 & 0.0002 & 8.57 & 21 \\
\hline ER_Philadelphia & 5.5131 & 0.0038 & 0.0002 & 8.62 & 20 \\
\hline ER_Anaheim & 3.8213 & 0.0640 & 0.0032 & 7.28 & 16 \\
\hline ER_Winnipeg & 4.8133 & 0.0184 & 0.0018 & 6.78 & 18 \\
\hline ER_Berlin & 4.8148 & 0.0066 & 0.0001 & 11.52 & 28 \\
\hline ER_Barcelona & 4.5363 & 0.0327 & 0.0013 & 7.35 & 18 \\
\hline ER_Terrassa & 5.9789 & 0.0537 & 0.0007 & 9.29 & 22 \\
\hline ER_Hessen & 4.0696 & 0.0379 & 0.0003 & 18.85 & 57 \\
\hline
\end{tabular}

ER graphs corresponding to the URNs with the same size, which are presented in Table 5. These ER graphs are generated by Pajek based on the $G(n, m)$ model, where $n$ denotes the number of nodes and $m$ represents the number of edges, and edges are picked uniformly at random. We can see from Table 5 that the average degree of ER graphs is larger than average in- and out-degree of the corresponding URNs in Table 3, which is expected since the ER graphs are undirected. In addition, average betweenness of most of the ER graphs is larger than that of the corresponding URNs, and diameter of ER graphs is smaller than that of the URNs in Table 3. These phenomena are probably caused by the random nature and weak connections of the ER graphs, which are more likely to form disconnected component clusters. Here, closeness centrality cannot be obtained due to weakly connected ER graphs. Based on the comparisons of $A P L$ and $C C$ between the URNs in Table 3 and the ER graphs in Table 5, these URNs cannot show significant small-world properties. The detailed analyses and discussions will be presented in the following section.
3.6. Small-Worldness of Urban Road Networks. Small-world effect was first mentioned by Karkubthy [42] and mathematically presented by Pool and Kochen [43]. The famous experiment related to small-worldness, "six degrees of separation," was conducted by Stanley Milgram in the 1960s [44]. Watts and Strogatz [3] constructed networks which lie between regular and random networks and named these "small-world" networks by analogy with Milgram's experiment. Small-world networks have $A P L$ as small as $A P L$ random, yet $C C \gg C C_{\text {random }}$ compared to random networks with the same number of nodes and edges, and a general feature of many complex networks is their small-world property [3], meaning that two nodes in the network can be connected with shorter paths. Following this, Newman [45] claimed that the value of the average shortest path for smallworld networks scales logarithmically or less with network size for a fixed mean degree. In order to explore whether these URNs have significant small-world properties, random networks which have the same number of nodes and the same average degree as these networks are generated using 
Pajek software so as to calculate their average path length $\left(A P L_{\text {random }}\right)$ and clustering coefficient $\left(C C_{\text {random }}\right)$. Here, it is worth noting that different formulas to approximate $A P L$ and $C C$ have been developed by many scholars, such as Newman et al. [46], Boccaletti et al. [47], and Albert and Barabási [48]. We also adopt commonly used formulas $(\log (n) / \log (\langle k\rangle))$ and $\langle k\rangle / n$ to approximate APL and CC, respectively. The comparisons between Pajek and these approximation formulas can be shown in Figure 7, which demonstrates that APL and CC based on these two methods are very similar and are of the same order of magnitude, although differences exist owing to randomness of ER graphs.

The comparisons between the generated random networks and the corresponding urban road networks are shown in Table 6.

As can be seen from Table 6, the APL of the nine reallife urban road networks (URNs) is much larger than that of the random networks with the same size. Although the $A P L$ of Anaheim is the closest to that of the corresponding random network, it still takes 4.5 more. In the meantime, most of the CCs for these nine URNs are larger than those for the corresponding random networks, but they are still in the same magnitude level, except for Berlin. The CCs of the URNs for Berlin and Winnipeg, meanwhile, are smaller than those for the corresponding random networks. In addition, the last column shows that $A P L$ does not scale logarithmically with the network size, $\operatorname{Ln}(N)$, where $N$ is the number of nodes. The above observations show that the small-world property is not significant among these nine URNs. In many studies of realistic networks, however, such as metabolic networks [49], the Chinese airport network [36], the Italian airport network [11], the worldwide air transportation network [10], the Boston subway network [37], the worldwide maritime transportation network [14], and public transport networks [16], small-world properties are evident. This may be due to the fact that these URNs are spatial and that all links are directional, which may increase the average geodesic distance between any node pairs and reduce the clustering effect in any given local area. This finding shows that these URNs are different from other networked transport systems in the real world, and this may imply more profound impacts of other characteristics of these networks.

\section{Correlation Analysis}

Many topological indices for the nine selected URNs have been calculated and analysed in Section 3. Based on this, a correlation between these topological indices can be conducted in order to explore the relationship between the topological indices and the underlying structural properties of these URNs. In addition, many interesting intuitive questions in the field of network science can be answered by correlation analyses of topological indices, for example, whether the most connected nodes are most central ones or those that are most compact at the local level [10].
4.1. Degree-Betweenness Correlation. The nodes with large degree are most connected, and the nodes with large betweenness are most central in the network. Nodes with large/ small betweenness and small/large degree are regarded as anomalies [10]. In fact, it is easy to design networks in which nodes have large betweenness and small degree. For example, if there are two communities connected by a single node with just two links, the node must have large betweenness due to the fact that it is frequently passed by routes which are going to one community from the other. This node plays an important role in such a network system. In order to check whether nodes with large degree have large betweenness for these URNs, this section calculates correlations between betweenness and in-degree and plots them as shown in Figure 8.

Many complex networks, such as the internet network [50], show that nodes with large betweenness also have a large degree, while the worldwide airport network presents anomalous behaviour in this regard [10]. As can be seen from Figure 8, the correlations between betweenness and indegree are weak, with neither presenting highly positive relationships nor showing anomalous behaviour, but instead exhibiting more complex relationships. Anaheim has the highest correlation, 0.62 , which means most of the city's nodes are well connected as well as central. Such relationships are almost nonexistent for Austin, Barcelona, Hessen, and Philadelphia, however, as shown by the low correlations (0.01, 0.16, 0.09, and 0.11, respectively). Such correlations may be caused by the distinct community characteristics of URNs. In addition, the correlations between out-degree and betweenness are identical to those between in-degree and betweenness, as shown in Figure 9.

4.2. Degree-Clustering Coefficient Correlation. In order to examine whether the well-connected nodes in these URNs still have a large clustering coefficient (CC), the correlations between $C C$ and in-degree are calculated and plotted, as shown in Figure 10. It is not surprising that the correlations between them are very low, in fact, all lower than 0.25 , since this is identical to the analyses of CC. For these URNs, the neighbouring nodes are less connected with each other. The correlations in the following confirm this viewpoint.

The correlations between $C C$ and out-degree are similar to those between $C C$ and in-degree (as shown in Figure 11) and against consistently very poor and lower than 0.25 . The reason why urban road networks with many links have such poor correlations is that these networks are directional and that the nodes in urban road networks tend to constitute quadrangles rather than triangles, which implies the neighbouring nodes of a given node are less likely to be connected with each other. This also explains why the clustering coefficients in these URNs are not very high.

\section{Communities of Urban Road Networks}

The previous sections have performed topological analysis, small-worldness, and correlation analysis between some topological indices. Building on these analyses, this section 


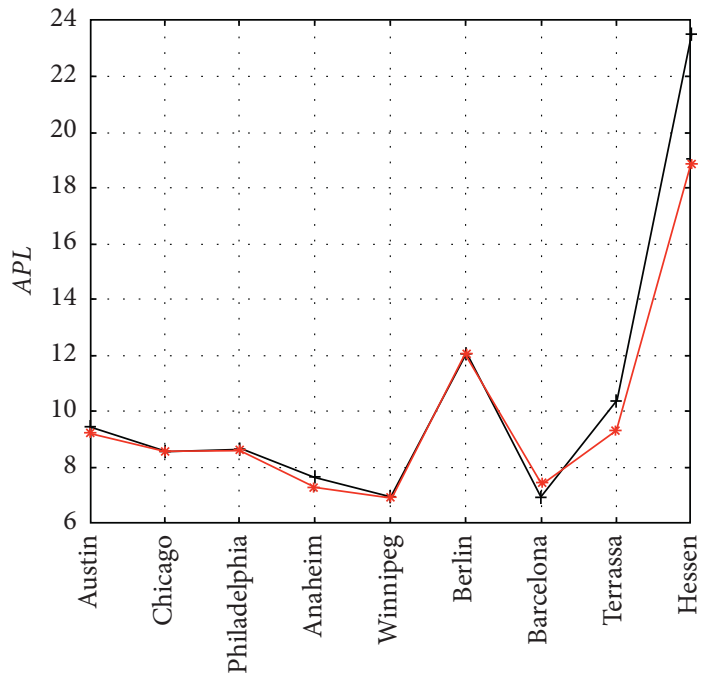

URNs $\leftarrow \log (n) / \log <k>$
$\rightarrow$ Pajek

(a)

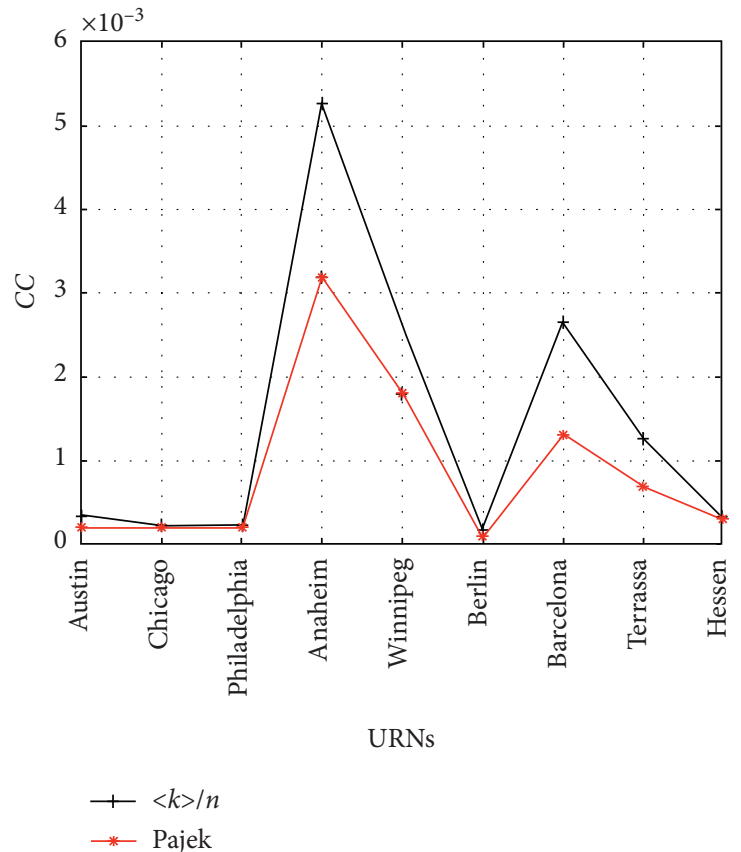

(b)

FIgURE 7: Comparisons between using Pajek and other approximation formulas.

TABle 6: $A P L$ and CC comparisons between nine URNs and the corresponding random networks of the same size.

\begin{tabular}{lccccc}
\hline City & $A P L$ & $C C$ & $A P L_{\text {random }}$ & $C C_{\text {random }}$ & $\operatorname{Ln}(N)$ \\
\hline Austin & 48.58 & 0.0010 & 9.2 & 0.0002 & 8.8940 \\
Chicago & 44.71 & 0.0017 & 8.6 & 0.0002 & 9.4713 \\
Philadelphia & 43.17 & 0.0007 & 8.6 & 0.0002 & 9.5022 \\
Anaheim & 11.87 & 0.0157 & 7.3 & 0.0032 & 6.0307 \\
Winnipeg & 18.85 & 0.0007 & 6.9 & 0.0018 & 6.9584 \\
Berlin & 50.09 & 0.0017 & 12 & 0.0001 & 9.4712 \\
Barcelona & 14.00 & 0.0008 & 7.4 & 0.0013 & 6.9276 \\
Terrassa & 25.09 & 0.0009 & 9.3 & 0.0007 & 7.3834 \\
Hessen & 45.60 & 0.0003 & 18.9 & 0.0003 & 8.4468 \\
\hline
\end{tabular}

detects and analyses community structures within these networks in order to explore the topological characteristics of the URNs further.

The detection and analysis of the community structures within large-scale networks have been a focus of research in recent years. The quantitative definitions of communities within networks are diverse. Fortunato [51] defined a community as groups of nodes which play a similar role or share common features or properties within those groups. Lancichinetti et al. [52] regarded "highly cohesive subgraph" of networks as communities, clusters, or modules. In biological and social networks [51], community structure is a common feature, and it is thought of as a division of networks into densely connected subgroups [53]. Lancichinetti et al. [52] proposed that a community is one pattern of node connections in realistic networks.

Detecting communities has many important applications in reality, such as identifying clusters of clients based on similar interests and geographical distance in order to provide better service [54], discovering communities of relationship networks between customers and online retailers so as to provide efficient purchase recommendations and marketing analyses [55], and identifying clusters in large networks for data storage [56]. In addition, the community structure of a network also offers a better way to understand networked systems more completely in that it is a powerful visualization tool to present the representation of networks rather than simply showing all the nodes and links of the network. In the transport field, in order to make clear the role of human collective behaviour phenomena across time and space in London transport, intertwined communities of traffic across the whole city were investigated by Petri et al. [57] to prove that this human spatial system is able to reach a self-organized critical state. Community detection has therefore been a hot topic in modern science of complex systems [51].

Many methods and algorithms can be applied to the detection of communities in graphs, such as graph partitioning, hierarchical clustering, and spectral clustering [51], but they all have limitations in handling edge weights, edge directions, or overlapping communities. In this study, Order Statistics Local Optimization Method (OSLOM), which is calculated based on the connectivity of networks and optimizes locally the statistical significance of clusters, is used to detect the community structure of these URNs. This was first proposed by Lancichinetti et al. [52], and it is capable of handling directed and weighted networks, overlapping community structure, hierarchical structure, pseudo-communities, and so on, due to the fact that OSLOM focuses on optimizing locally the statistical significance of clusters. In 


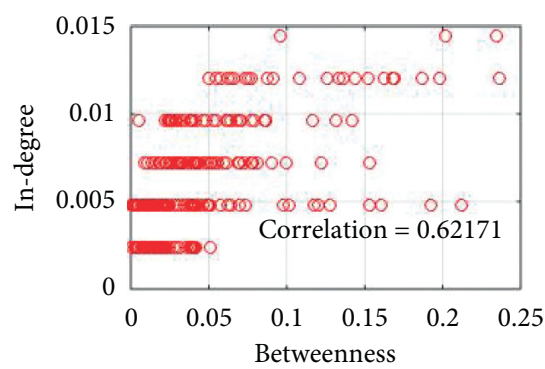

(a)

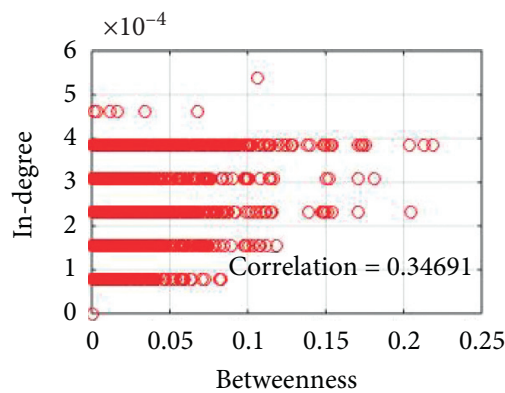

(d)

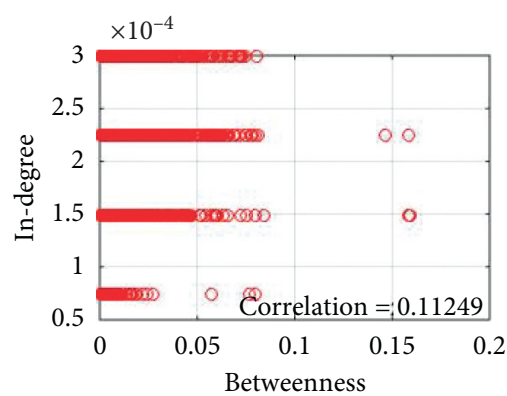

(g)

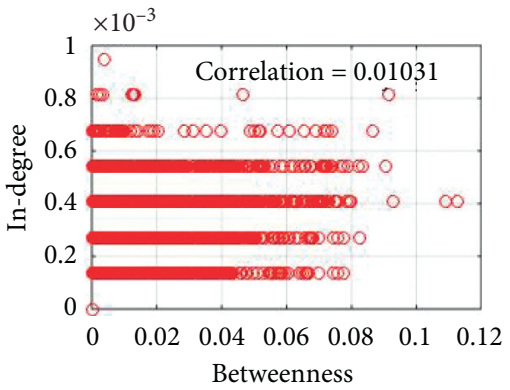

(b)

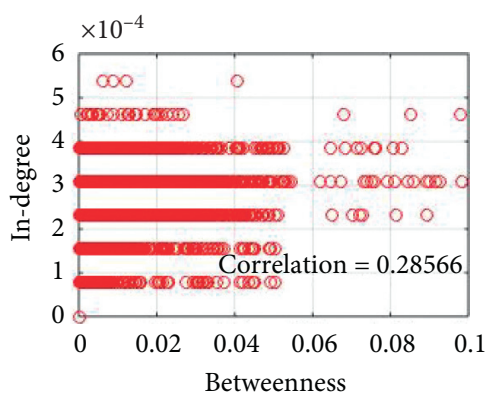

(e)

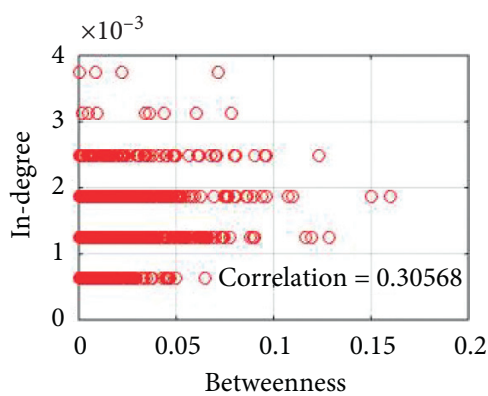

(h)

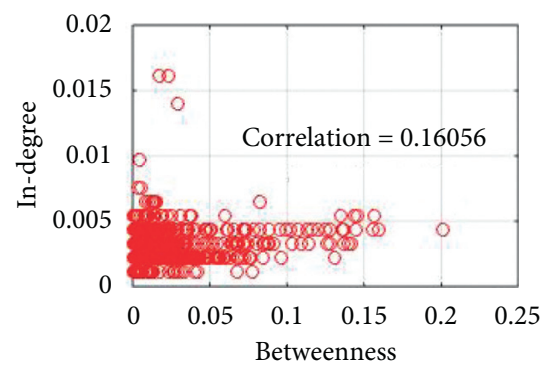

(c)

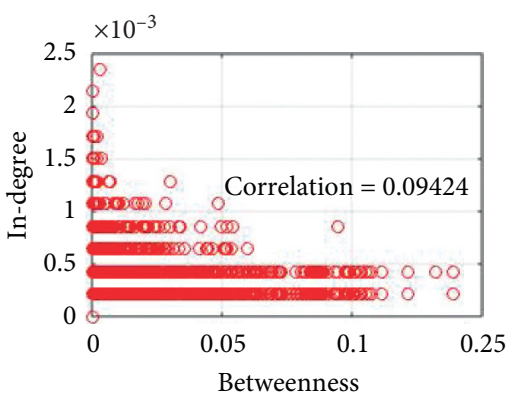

(f)

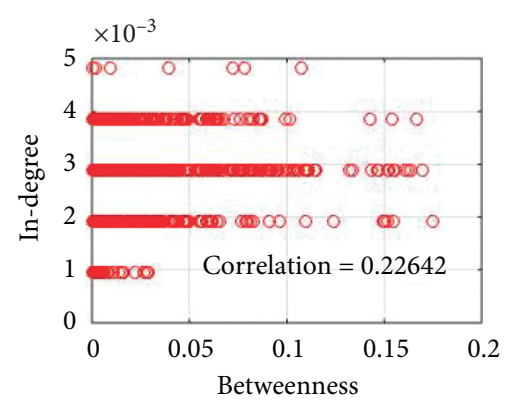

(i)

Figure 8: The correlation between normalized in-degree and betweenness: (a) Anaheim; (b) Austin; (c) Barcelona; (d) Berlin; (e) Chicago; (f) Hessen; (g) Philadelphia; (h) Terrassa; (i) Winnipeg.

order to better understand how OSLOM works for detection of communities and hierarchies, we present the core algorithm in equation (1). Suppose $\varphi$ is a graph with $N$ nodes and $S$ links and $\vartheta$ is a subgraph of graph $\varphi$ generated by the configuration model, which can be referred to Lancichinetti et al. [58]. The probability of node $i$ with $k_{i}^{\text {in }}$ neighbors in subgraph $\vartheta$ can be written as

$$
p\left(k_{i}^{\text {in }} \mid i, \vartheta, \varphi\right)=A \frac{2^{-k_{i}^{\text {in }}}}{k_{i}^{\text {out }} ! k_{i}^{\text {in }} !\left(m_{c}^{\text {out }}-k_{i}^{\text {in }}\right) !\left(M^{*} / 2\right) !},
$$

where $m_{c}^{\text {out }}$ is the out-degree of the subgraph $\vartheta, k_{i}^{\text {in }}$ and $k_{i}^{\text {out }}$ are in- and out-degree of node $i$, respectively, $M^{*}$ denotes the internal degree of the rest of nodes $\varphi /[\vartheta \cup\{i\}]$, and $A$ is a normalization factor. This equation may enumerate the possible configurations of the network with $k_{i}^{\text {in }}$ between node $i$ and $\vartheta$. Based on this equation, OSLOM is able to find external significant vertices and include them into $\vartheta$, and it repeats this procedure to look for significant clusters until convergence; following this, OSLOM analyses the resulting set of clusters to check their internal structure and possible unions. To analyse the hierarchical structure, we need to build a supernetwork, where nodes refer to clusters and links exist if the clusters are connected; then, OSLOM is employed for this supernetwork so as to obtain the second hierarchical layer, which can also be regarded as a new supernetwork, and the above procedure is repeated until no clusters can be generated. More details concerning the detection of communities and hierarchical structure are referred to Lancichinetti et al.'s work [52].

As a lifeline infrastructure, URNs can be regarded as one type of self-organized system, which means that they are similar to other systems in nature to some extent. In order to examine this natural property, community detection and analyses are conducted based on the OSLOM, and the results are shown in Figure 12.

The communities of all nine URNs are presented in Figure 12. The first impression of these communities is that the bigger the URNs, the more the complex community 


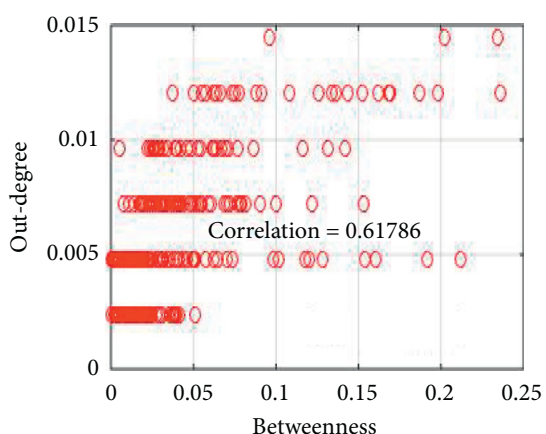

(a)

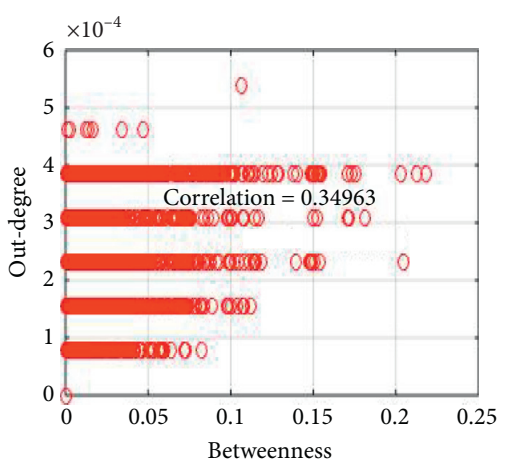

(d)

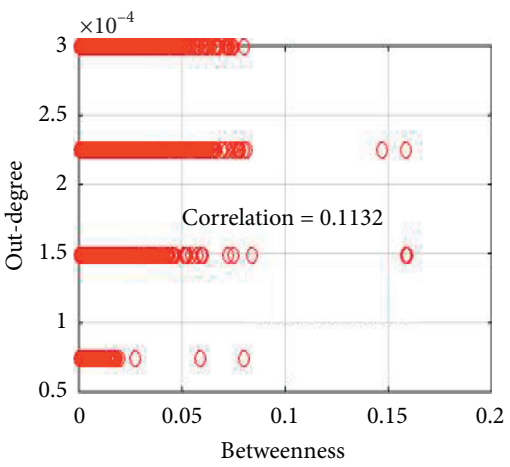

(g)

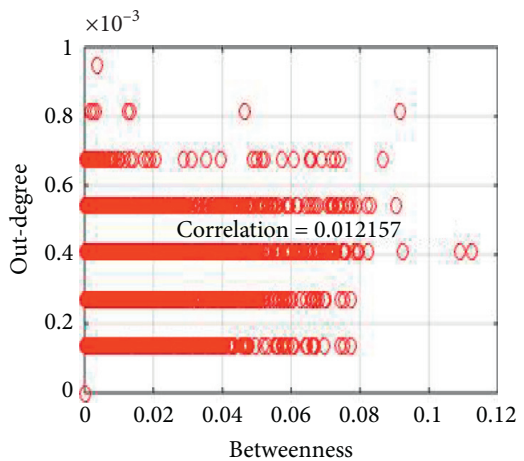

(b)

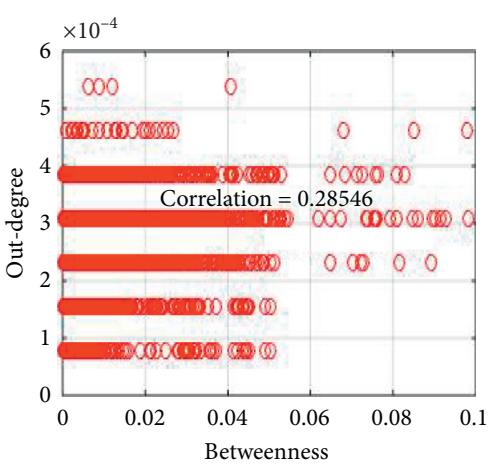

(e)

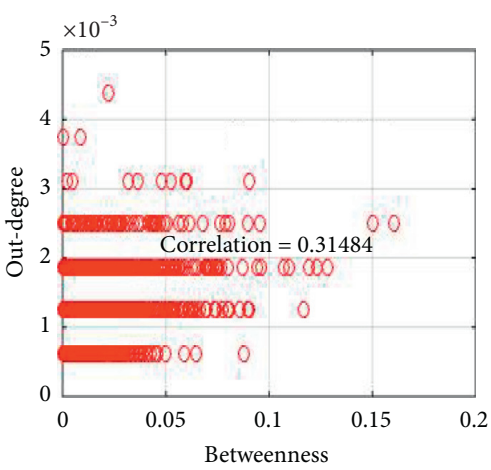

(h)

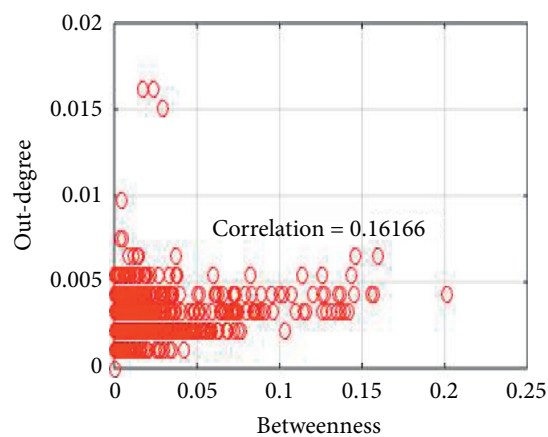

(c)

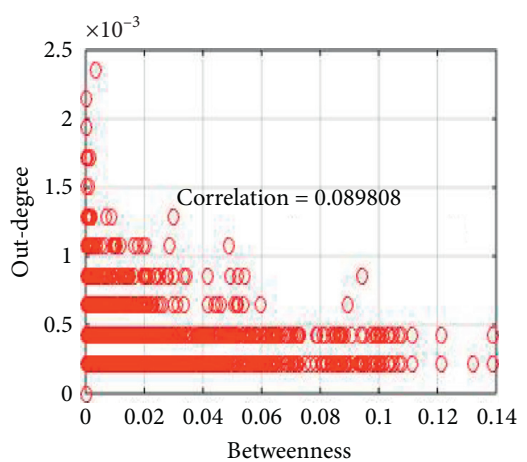

(f)

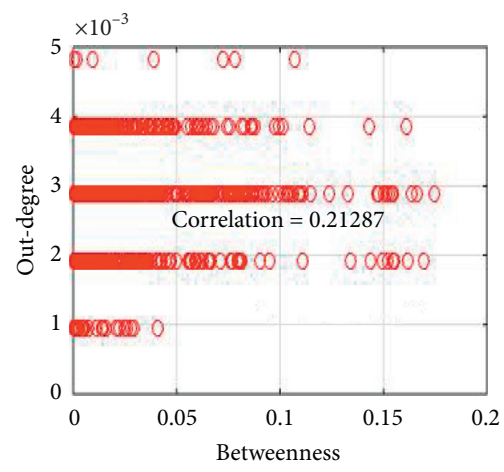

(i)

Figure 9: The correlation between normalized out-degree and betweenness: (a) Anaheim; (b) Austin; (c) Barcelona; (d) Berlin; (e) Chicago; (f) Hessen; (g) Philadelphia; (h) Terrassa; (i) Winnipeg.

structure they have. For example, Austin, Berlin, Chicago, and Philadelphia are the larger networks, and the number of communities for these URNs is apparently more than that in the smaller networks. In addition, it seems that these bigger URNs have a significant hierarchical structure, namely, the bigger clusters consist of several smaller ones. The smaller networks, such as Anaheim, Terrassa, and Winnipeg, meanwhile, present a clearer community structure. In addition, some nodes which look like not belonging to any communities in these URNs are overlapping ones, which suggests that these nodes are shared by several communities, and the nodes may assist the mutual communications for these communities; thus, these "sharing nodes" may have great impact on the robustness and vulnerability of the whole network.
In this study, the community detection is mainly based on the estimation of the statistical significance of clusters and connectivity of the network. Although it seems that the size of communities is likely to be correlated with other communities of the URNs due to the fixed number of nodes and links, the quantitative correlations between communities of the URNs are unclear. As the developers of the OSLOM, Lancichinetti et al. [52] did not claim the existence of such correlations either, but we may explore it for the future study. Here, we are only able to confirm that the communities within a given URN can be connected with other ones of the URN by the overlapping nodes or some nodes within these communities, which further highlights the importance of such nodes in communication between communities. 


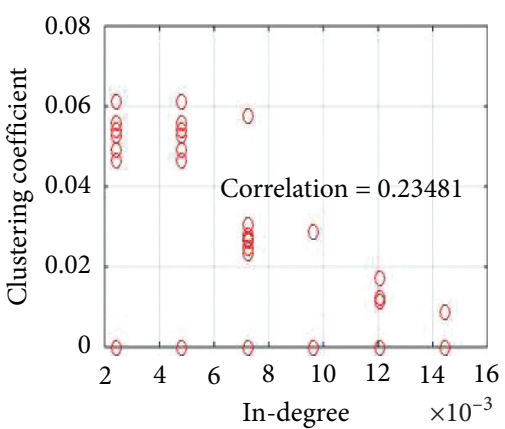

(a)

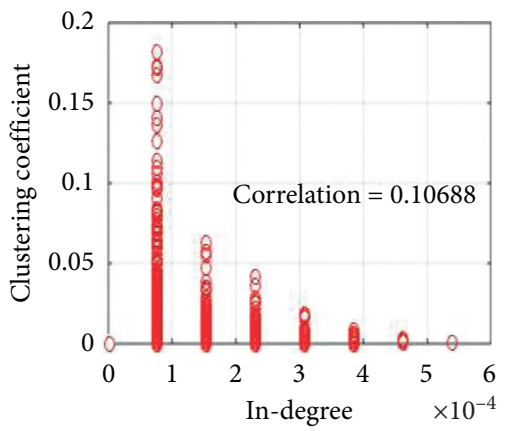

(d)

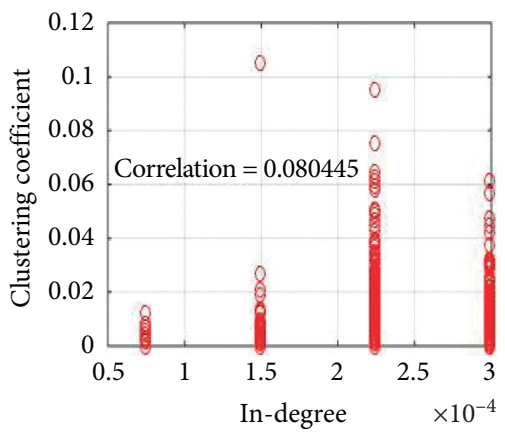

(g)

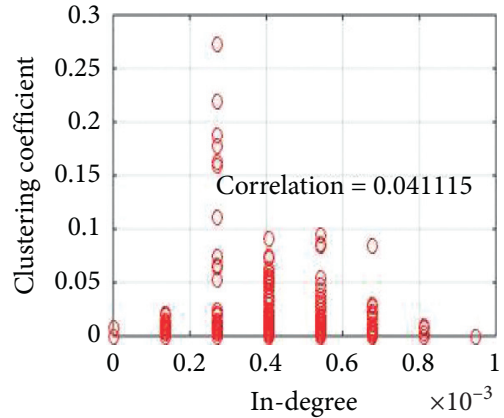

(b)

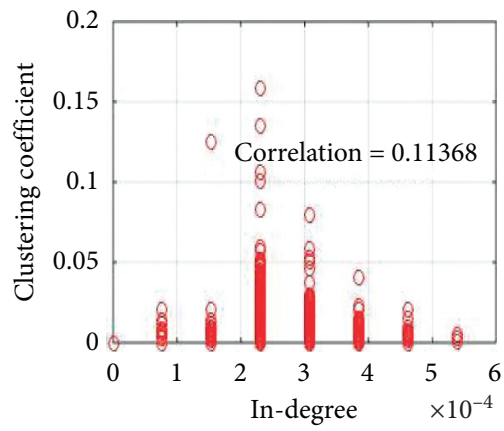

(e)

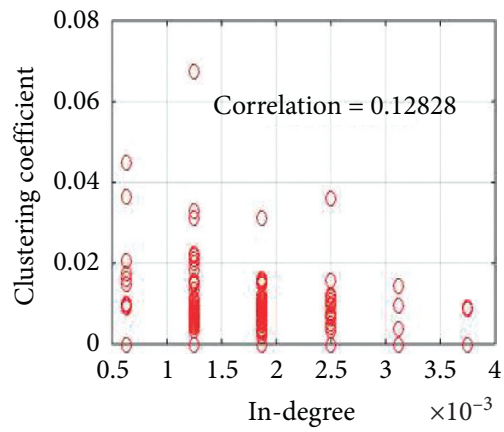

(h)

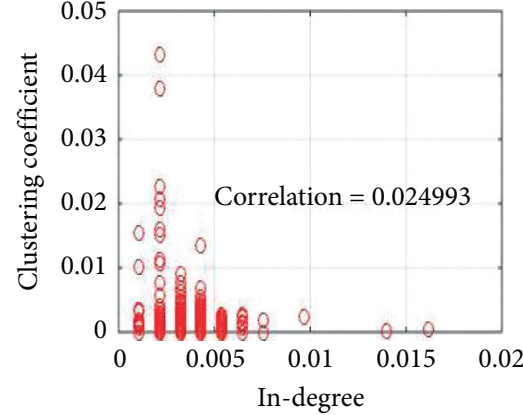

(c)

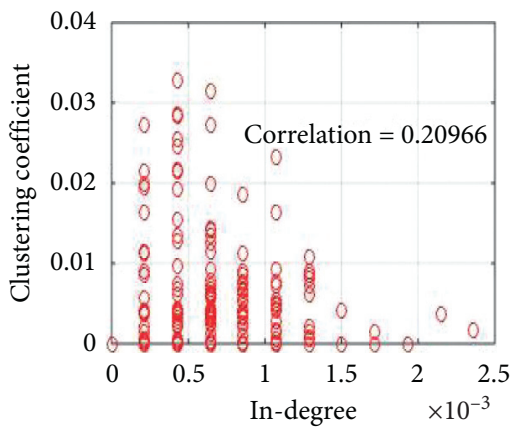

(f)

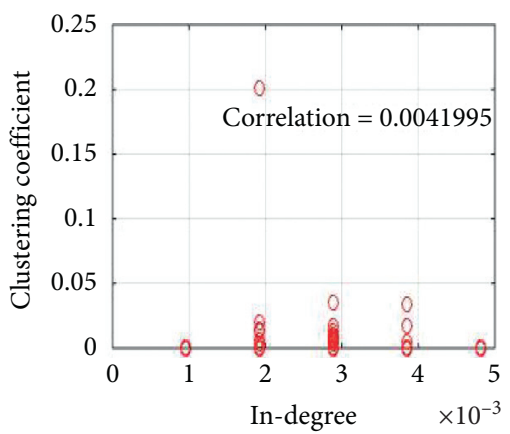

(i)

FIgURE 10: The correlation between normalized in-degree and clustering coefficient: (a) Anaheim; (b) Austin; (c) Barcelona; (d) Berlin; (e) Chicago; (f) Hessen; (g) Philadelphia; (h) Terrassa; (i) Winnipeg.

In addition, we need to emphasize that exploration of the relationship between the community structure of URNs and their spatial distribution is highly important. However, since the data source used in this study lacks data related to the geographic information, such as the node coordination and locations of urban functional areas, the study concerning the spatial distribution of communities is very difficult to be conducted, and we also cannot distinguish the areas in real cities based on the communities inside hierarchical layers of the URNs without realistic geographic information data.

The number of hierarchical layers and the number of communities in each hierarchical layer are presented in Table 7.

Identifying the modules and structure of the hierarchy using the topology information encoded in the networks is the main purpose of detecting communities [51]. As can be seen from Table 7, larger URNs are more likely to have more communities and hierarchical layers. For example, Philadelphia, Berlin, and Chicago have 6, 5, and 5 hierarchy layers, respectively, and the number of communities in the lowest layers is 343, 457, and 266, respectively. Anaheim, with just 416 nodes, however, has just two hierarchical layers, and the number of communities at the lowest layer is just 11. Due to the fact that the detection of the hierarchical structure is mainly based on repeatedly using the OSLOM for the construction of supernetworks, where nodes refer to clusters and links exist if the clusters are connected, we may say that the number of hierarchical layers represents the depth of the network structure, and lowest and highest hierarchical layers indicate the microcommunities and the macrocommunities, respectively. For example, Lancichinetti et al. [52] utilized the OSLOM for the UK commuting network; three hierarchical layers are detected, and the highest hierarchical layer represents the four recognizable countries: England, Wales, Scotland, and Northern Ireland.

Here, due to the limitation of the data source, we only conduct the spatial analysis for the Anaheim network. Based 


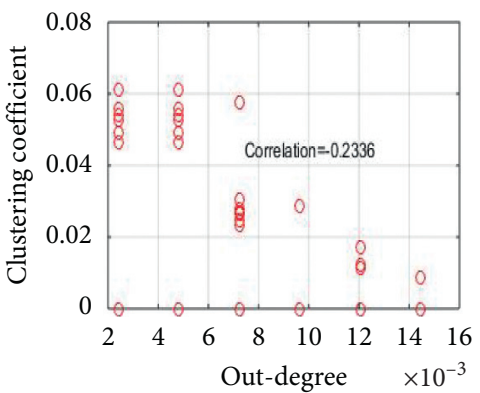

(a)

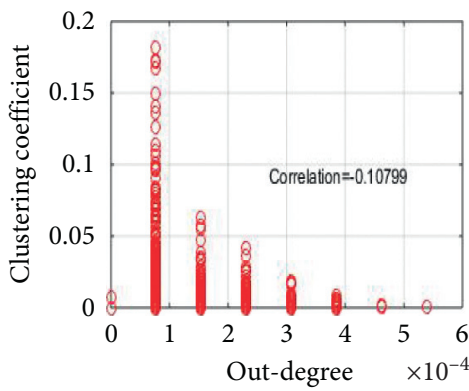

(d)

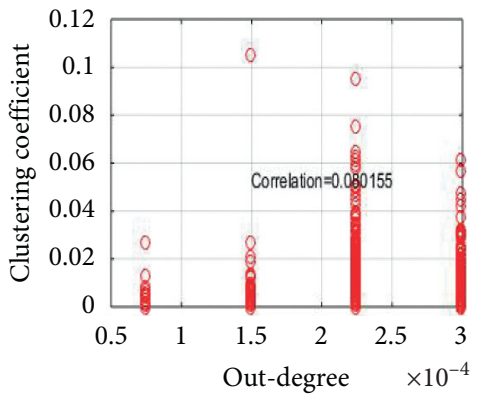

(g)

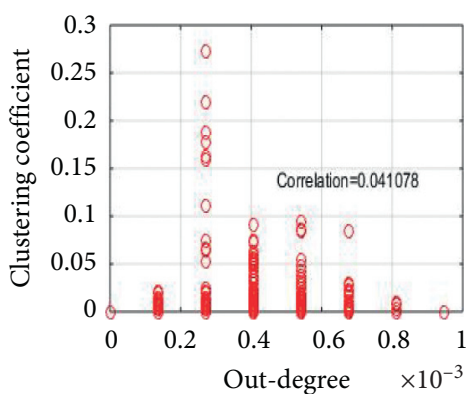

(b)

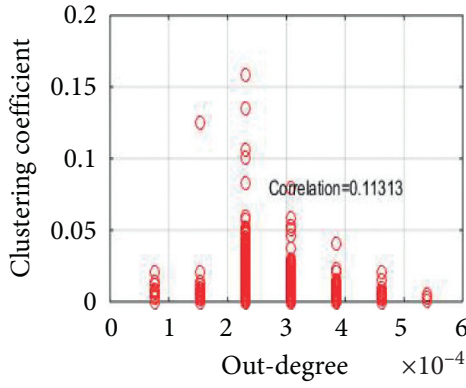

(e)

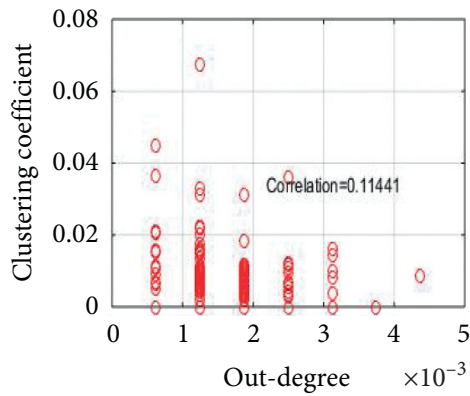

(h)

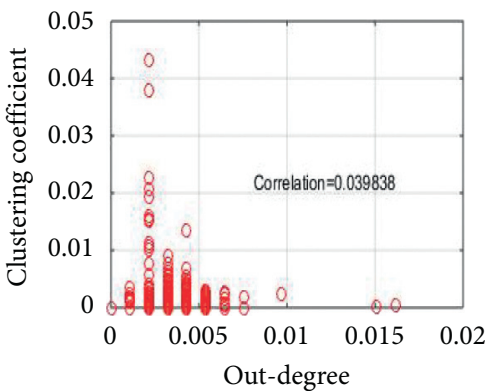

(c)

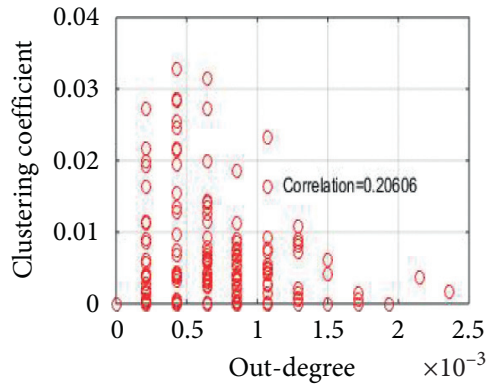

(f)

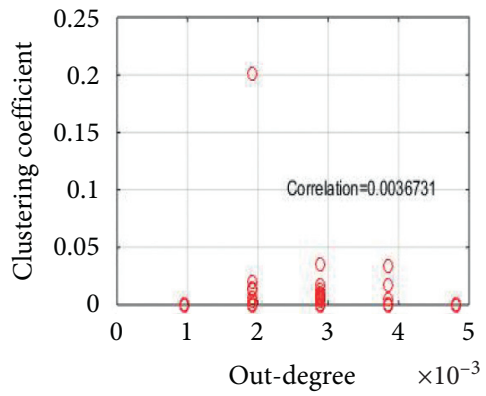

(i)

FIGURE 11: The correlation between normalized out-degree and clustering coefficient: (a) Anaheim; (b) Austin; (c) Barcelona; (d) Berlin; (e) Chicago; (f) Hessen; (g) Philadelphia; (h) Terrassa; (i) Winnipeg.

on the coordinate information, we plot the network with QGIS, which is presented as follows.

As can be seen from Figure 13, the distribution of communities at different layers is closely related to the locations of nodes of the Anaheim network. For example, the nodes (green) at the northeast corner tend to form community 1 at layer 1 ; in addition, from a broader perspective, the nodes (diamond) across the entire northeastern region constitute community 2 at layer 2 . Here, layers 1 and 2 represent the depth of the network structure. Because the algorithm used for community detection is based on the connectivity, the close relationship between the distribution of communities and hierarchies and the location of nodes is expected. However, we still do not know whether these communities are related to the layout of urban distinguished/functional areas due to the lack of realistic geographical information data, such as locations of hospitals, schools, and commercial centers. In the future, OpenStreetMap (OSM) and Geographic Information System (GIS) can be utilized to explore the relationship between the topology of URNs and realistic geographical information, such as correlations between topological characteristics and points of interests (POI), connections between community, hierarchy structure, and distinguished areas within cities.

In order to more clearly present the spatial distribution of communities, we also use MATLAB to plot the Anaheim network, as shown in Figure 14.

Since all these URNs consist of many communities, with each community consisting of many individual nodes, the size of communities varies. To some extent, these communities may follow a certain distribution rule. In order to identify this point, histograms can be plotted, as shown in Figure 15.

As can be seen from Figure 15, the larger URNs, such as Austin, Berlin, and Chicago, tend to follow a normal distribution. Among these URNs, the left side of figures regarding Austin, Berlin, and Chicago does not fit well with the normal distribution compared to their right side. The red lines in the graphs are the normal distribution fitting lines and clearly identify this point. $R^{2}$, meanwhile, which is 


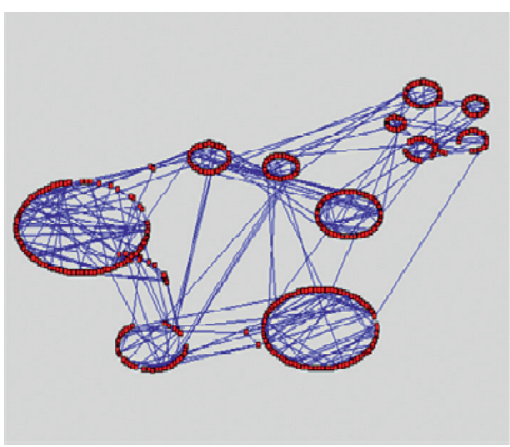

(a)

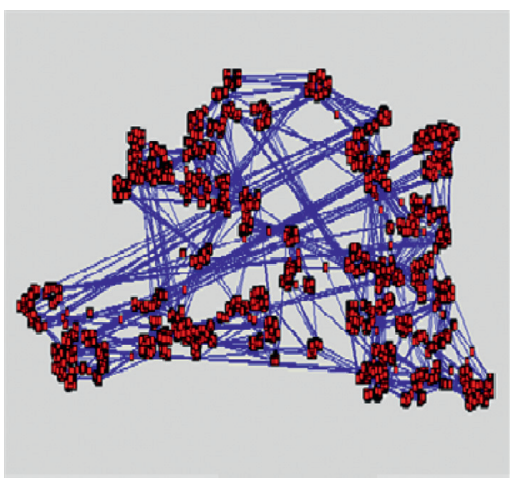

(d)

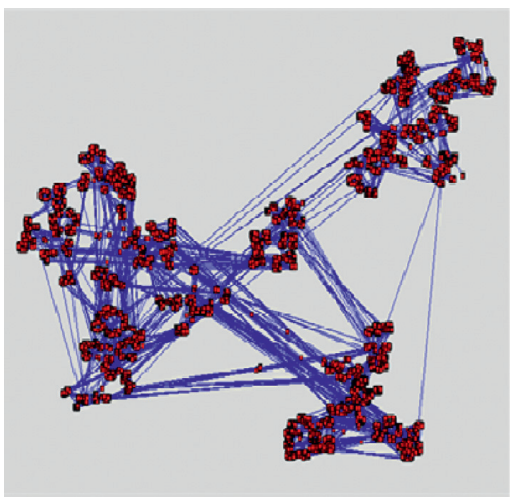

(g)

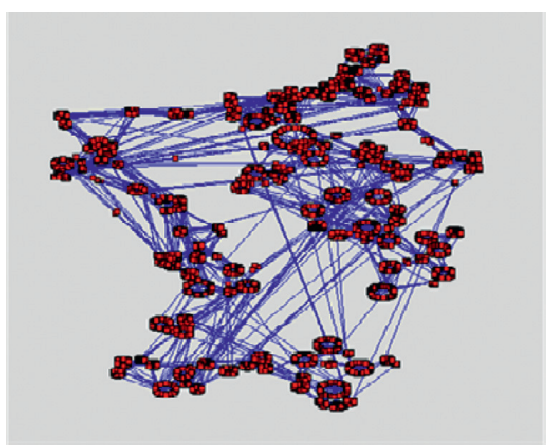

(b)

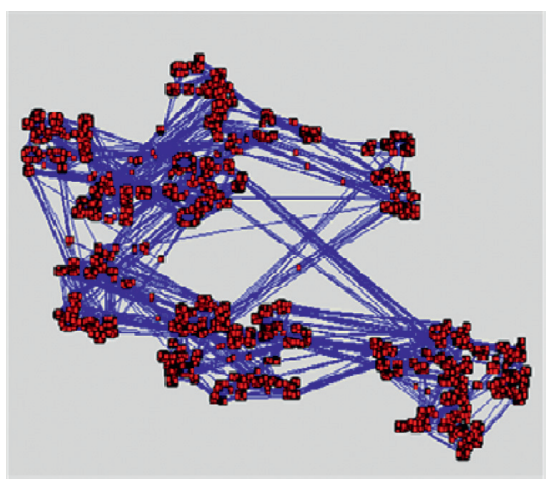

(e)

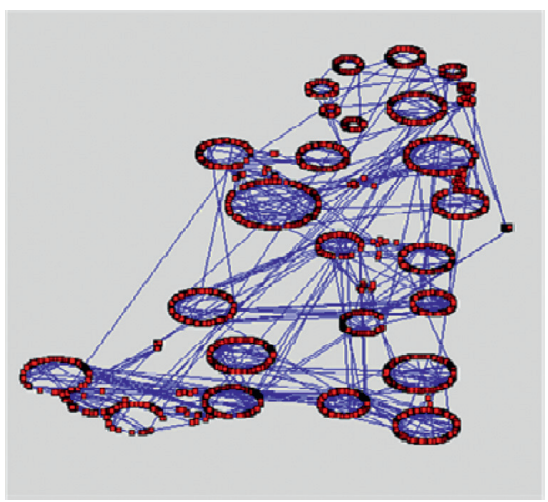

(h)

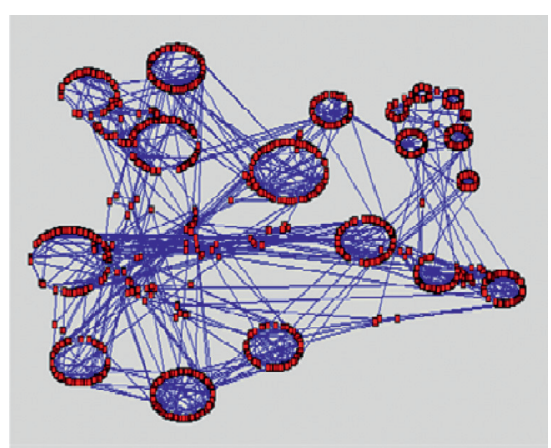

(c)

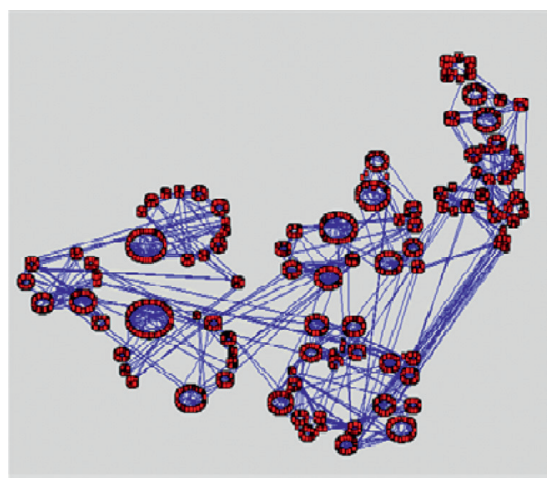

(f)

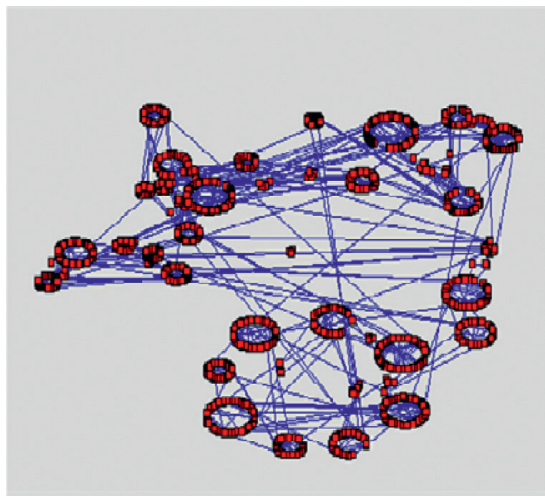

(i)

Figure 12: Schematic graphs of communities for nine urban road networks: (a) Anaheim; (b) Austin; (c) Barcelona; (d) Berlin; (e) Chicago; (f) Hessen; (g) Philadelphia; (h) Terrassa; (i) Winnipeg.

TAвLE 7: Structure of hierarchy and communities in each hierarchical layer for nine URNs.

\begin{tabular}{lccc}
\hline City & Nodes & Hierarchy layers & Communities in each hierarchy (from low to high) \\
\hline Austin & 7388 & 5 & $151 ; 46 ; 13 ; 7 ; 5$ \\
Chicago & 12,982 & 5 & $266 ; 81 ; 22 ; 7 ; 2$ \\
Philadelphia & 13,389 & 6 & $343 ; 100 ; 29 ; 11 ; 6 ; 3$ \\
Anaheim & 416 & 3 & $11 ; 2$ \\
Winnipeg & 1052 & 5 & $30 ; 8 ; 7$ \\
Berlin & 12,981 & 3 & $457 ; 112 ; 34 ; 14 ; 5$ \\
Barcelona & 1020 & 3 & $20 ; 6 ; 4$ \\
Terrassa & 1609 & 4 & $27 ; 5 ; 3$ \\
Hessen & 4660 & & $104 ; 25 ; 8 ; 5$ \\
\hline
\end{tabular}

calculated based on the maximum likelihood method, represents the goodness of the communities of the URNs following normal distribution. Conversely, those URNs with a smaller size apparently have worse normal distribution, and it seems that Anaheim and Winnipeg do not follow any distribution rules due to the fact that these URNs are relatively small, so 


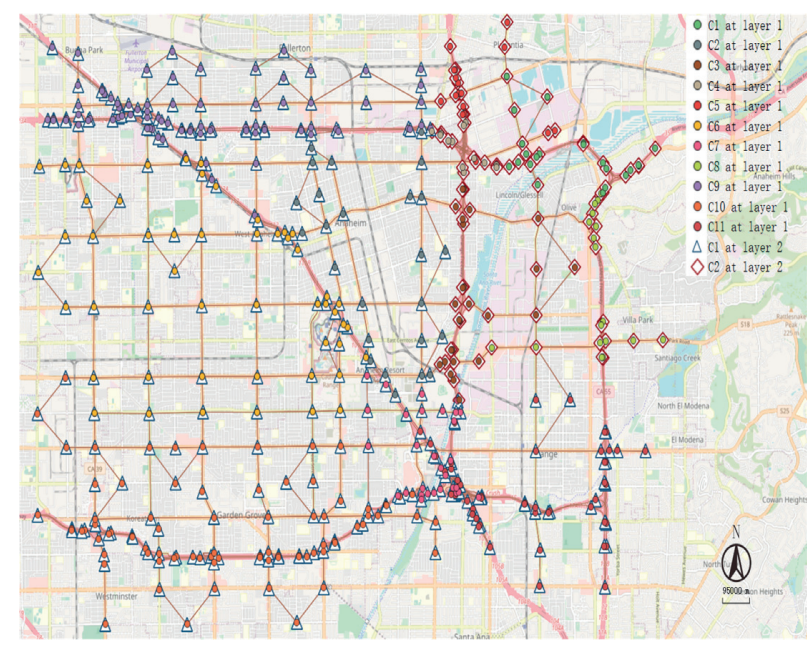

Figure 13: Communities and hierarchies of the Anaheim network plotted by QGIS.

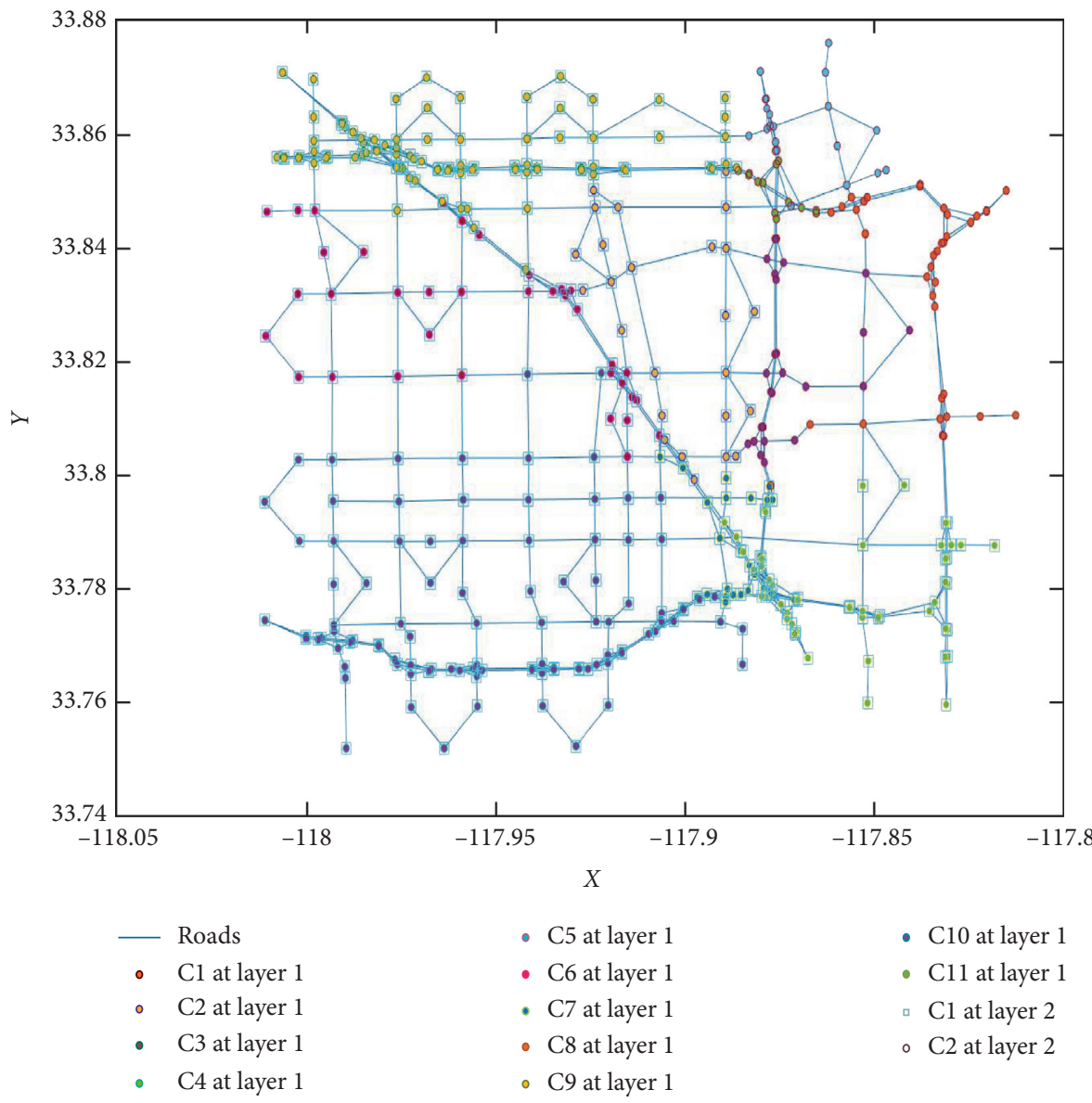

Figure 14: Communities and hierarchies of the Anaheim network plotted by MATLAB.

there are not too many hierarchical layers and communities. Through the above analysis, a rough conclusion is able to be summarised; that is, the larger the URNs are, the better the normal distribution fitting they have.

The detection of communities for URNs may provide insightful views for the management and planning of transport within urban areas. For example, installing detectors, sensors, or any other electronic facilities based on the distribution of communities may efficiently collect data such as traffic flows, vehicle speed, and traffic incidents to improve network safety or mitigate traffic congestion. In addition, identification of "sharing nodes" is helpful for 


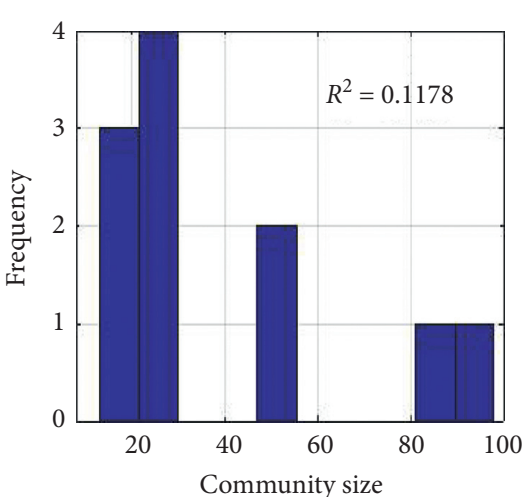

(a)

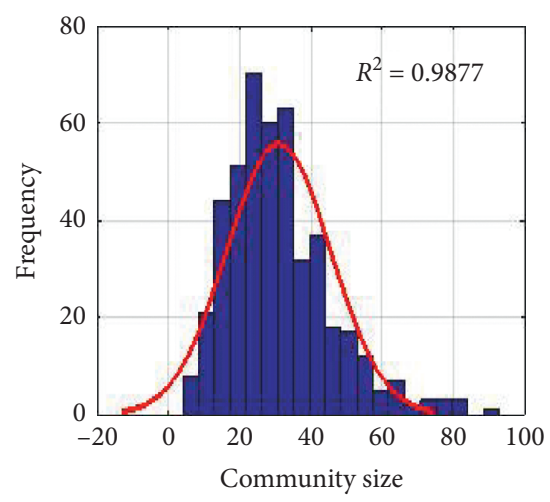

(d)

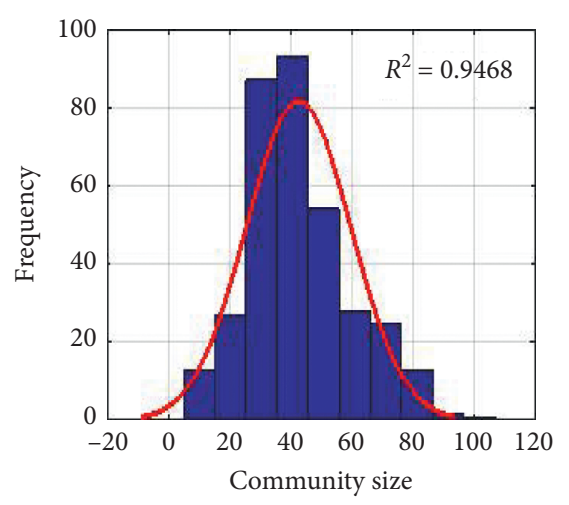

(g)

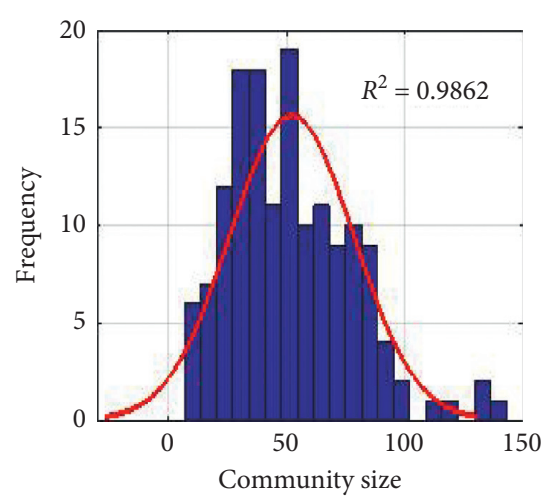

(b)

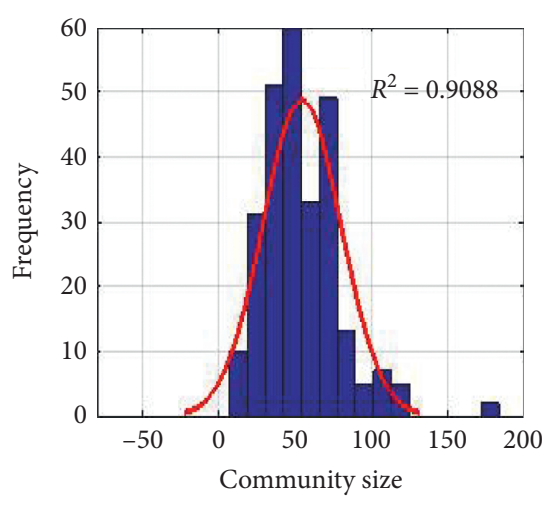

(e)

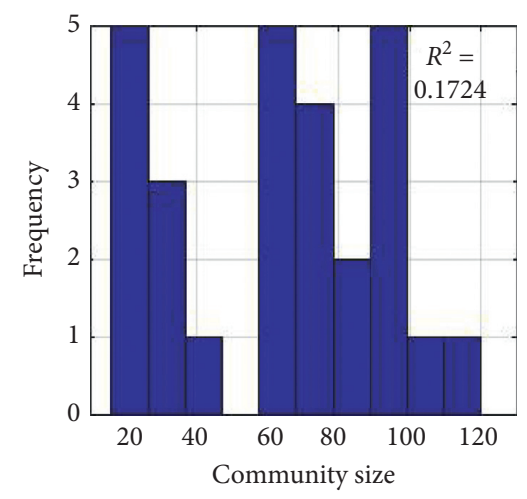

(h)

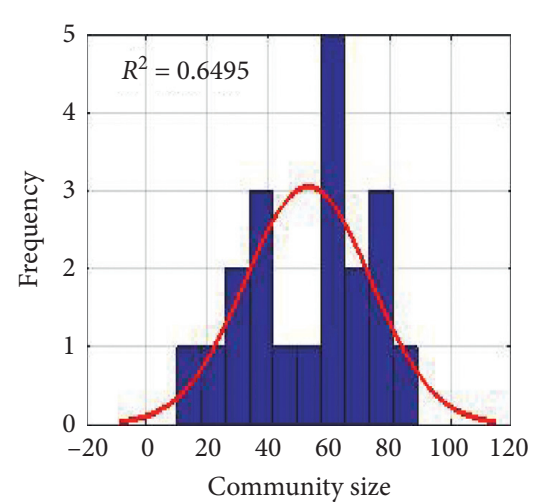

(c)

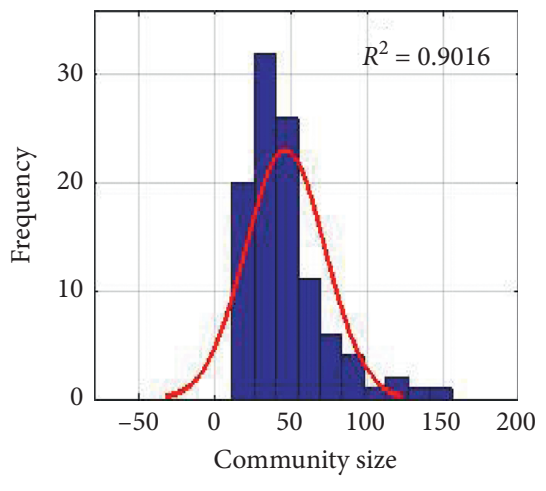

(f)

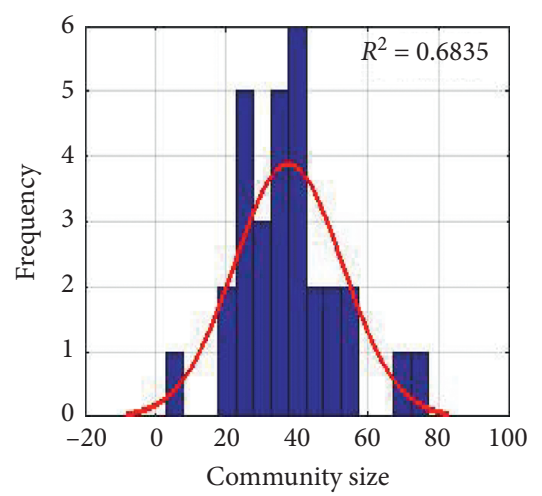

(i)

Figure 15: Histograms for nine URNs: (a) Anaheim; (b) Austin; (c) Barcelona; (d) Berlin; (e) Chicago; (f) Hessen; (g) Philadelphia; (h) Terrassa; (i) Winnipeg.

protecting critical parts of the systems and maintaining robustness of URNs. Furthermore, through the detection and analysis of communities and hierarchies of URNs, we may say that the URNs also tend to follow general nature rules in natural systems [59], such as a hierarchical structure and a normal distribution.

\section{Conclusions}

As technologies related to smart city and cooperative vehicle infrastructure systems (CVIS) are increasingly prevailing, urban roads and their constituent urban road networks receive more and more attention. In this context, this paper has investigated the topological characteristics of a number of URNs for the first time in terms of purely physical roads. Widely used topology indices based on complex network theory were utilized in this paper to explore the topological characteristics of the nine URNs, small-world analysis and correlation analysis between some topological indices are conducted, and relatively new notions such as community detection were also applied to supplement the understanding of these topological networks. The results can be summarised as follows:

(1) The average in-degree and average out-degree of these URNs are exactly the same, and the distributions of in-degree and out-degree are very similar, 
despite the fact that these URNs are directional. In addition, the distributions of in-degree and outdegree for these URNs follow the power law, suggesting that most of the nodes have small in-degree and out-degree, which means the URNs overall are less well connected. Philadelphia is overall less well connected than the other URNs.

(2) The clustering coefficients (CC) of the URNs are very low, showing the URNs are less compact in local areas. The average CC of these URNs is smaller compared to other transport networks.

(3) Most of the weighted closeness of these URNs also follows an exponential distribution, and Anaheim and Berlin are the URNs in which most of the nodes have worse accessibility to other nodes.

(4) The weighted betweenness centrality (WBC) of all URNs follows a power-law distribution well, and parameter $\lambda\left(P(B C \geq x) \propto x^{-\lambda}\right)$ for the distributions is between 0.4 and 0.8 .

(5) APL and diameters are proportional with the size of the URNs, and efficiency measures are consistent with APL. The results show that Berlin has the worst efficiency, while Anaheim has the best efficiency in terms of APL.

(6) Unlike other networked systems, such as metabolic networks and transport networks, these URNs do not have significant small-world properties.

These results reveal that URNs have distinctive statistical characteristics compared to other transport networks, such as the Chinese airport network [36], the Italian airport network [11], the worldwide air transportation network [10], and the Boston subway network [37]; particularly, these URNs do not have significant small-worldness, which may be caused by the unique spatial properties of urban roads.

Based on this, the relationships among in-degree, outdegree, $W B C$, and $C C$ have been examined, and the results reveal that the correlations between degree, $W B C$, and $C C$ are not significant; neither highly positive relationships nor anomalies exist in these URNs.

Following this, community detections for the URNs were conducted using the OSLOM, and the results show that the larger the URNs were, the more communities and hierarchical layers they had. In addition to this, the distributions of communities at the lowest hierarchical layer for the URNs tend to fit a normal distribution better if the URNs are larger. These findings are compatible with common phenomena in natural and social science [59]. The community detection of the URNs will facilitate the detector installation, data collection, and identification of critical parts of cities.

The purpose of this study has been to explore the unique characteristics of the URNs. This work also helps to prepare the ground for further study of other important properties of URNs, such as robustness and resilience. The topological indices shown in this study are usually related to the robustness of networks due to the fact that some of these measures are able to reflect the efficiency and functionality of those networks $[33,41,60,61]$, and this feature can be used for the assessment of robustness and resilience combining the operational data of URNs, such as the traffic flow and road capacity.

Due to the fact that the data sources related to URNs are very limited, in this study, the URNs are mainly located in Europe and North America, such as Chicago, Austin, Berlin, and Barcelona. In the future, with data sourced from other parts of the world, in particular from less developed countries or regions, it will be possible to expand our comparisons to a wider context. In particular, it is hypothesized that the diversity in social, economic, cultural, and geographical characteristics could manifest itself in the formation of URNs and thus may be reflected in the topological features of these URNs. Future work along these lines may uncover qualitatively and quantitatively different topological or operational features of URNs from a geographically representative set of networks. In addition, recently, we have noticed that OpenStreetMap (OSM) may provide abundant raw data for many city networks, which include all types of roads and require to be processed to satisfy specific study requirements. In the future, we may utilize OSM data to analyse the common topological characteristics and community structure of URNs across the world.

\section{Data Availability}

The data of urban road networks used to support this study are available from the database website http://www.bgu.ac.il/ $\sim$ bargera/tntp/.

\section{Conflicts of Interest}

The authors declare that there are no conflicts of interest regarding the publication of this paper.

\section{Acknowledgments}

This work was supported in part by the Chinese Scholarship Council (Grant no. 201308060082) and Key Special Project of Beijing City (Grant no. Z18110000391801).

\section{References}

[1] W. L. Shang, "Robustness and resilience analysis of urban road networks," Ph.D. Thesis, Imperial College London, London, UK, 2017.

[2] S. Goh, M. Y. Choi, K. Lee, and K.-m. Kim, "How complexity emerges in urban systems: theory of urban morphology," Physical Review E, vol. 93, no. 052309, pp. 1-12, 2016.

[3] D. J. Watts and S. H. Strogatz, "Collective dynamics of "smallworld” networks," Nature, vol. 393, no. 6684, pp. 440-442, 1998.

[4] A.-L. Barabási and R. Albert, "Emergence of scaling in random networks," Science, vol. 286, no. 5439, pp. 509-512, 1999.

[5] C. Ducruet and L. Beauguitte, "Spatial science and network science: review and outcomes of a complex relationship," Networks and Spatial Economics, vol. 14, no. 3-4, pp. 297-316, 2014. 
[6] C. Von Ferber, T. Holovatch, Y. Holovatch, and V. Palchykov, "Public transport networks: empirical analysis and modeling," The European Physical Journal B, vol. 68, no. 2, pp. 261-275, 2009.

[7] S. D. Dimitrov and A. Ceder, "A method of examining the structure and topological properties of public-transport networks," Physica A: Statistical Mechanics and Its Applications, vol. 451, pp. 373-387, 2016.

[8] Y. Korduba, Y. Holovatch, and R. De Regt, "Physicist's approach to public transportation networks: between data processing and statistical physics," Annales Universitatis Mariae Curie-Sklodowska, Sectio A-Mathematica, vol. 73, no. 2, pp. 69-89, 2020.

[9] C. Von Ferber, Y. Holovatch, and V. Palchykov, "Scaling in public transport networks," Condensed Matter Physics, vol. 8, no. 1, pp. 225-234, 2005.

[10] R. Guimera, S. Mossa, A. Turtschi, and L. A. N. Amaral, "The worldwide air transportation network: anomalous centrality, community structure, and cities' global roles," Proceedings of the National Academy of Sciences, vol. 102, no. 22, pp. 7794-7799, 2005.

[11] M. Guida and F. Maria, "Topology of the Italian airport network: a scale-free small-world network with a fractal structure?" Chaos, Solitons \& Fractals, vol. 31, no. 3, pp. 527-536, 2007.

[12] W. Li and X. Cai, "Empirical analysis of a scale-free railway network in China," Physica A: Statistical Mechanics and Its Applications, vol. 382, no. 2, pp. 693-703, 2007.

[13] L. Guo and X. Cai, "Degree and weighted properties of the directed China railway network," International Journal of Modern Physics C, vol. 19, no. 12, pp. 1909-1918, 2008.

[14] Y. Hu and D. Zhu, "Empirical analysis of the worldwide maritime transportation network," Physica A: Statistical Mechanics and Its Applications, vol. 388, no. 10, pp. 20612071, 2009.

[15] P. Kaluza, A. Kölzsch, M. T. Gastner, and B. Blasius, "The complex network of global cargo ship movements," Journal of The Royal Society Interface, vol. 7, pp. 093-103, 2010.

[16] J. Sienkiewicz and J. Hołyst, "Statistical analysis of 22 public transport networks in Poland," Physical Review E, vol. 72, no. 4, 2005.

[17] X. Xu, J. Hu, F. Liu, and L. Liu, "Scaling and correlations in three bus-transport networks of China," Physica A: Statistical Mechanics and Its Applications, vol. 374, no. 1, pp. 441-448, 2007.

[18] X. Zheng, J. Chen, and J. Shao, "Analysis on topological properties of Beijing urban public transit based on complex network theory," Acta Physica Sinica, vol. 61, Article ID 190510, 2012.

[19] S. Derrible, "Network centrality of metro systems," PLoS One, vol. 7, Article ID e40575, 2012.

[20] L. Sun, Y. Huang, Y. Chen, and L. Yao, "Vulnerability assessment of urban rail transit based on multi-static weighted method in Beijing, China," Transportation Research Part A: Policy and Practice, vol. 108, pp. 12-24, 2018.

[21] R. De Regt, C. von Ferber, Y. Holovatch, and M. Lebovka, "Public transportation in Great Britain viewed as a complex network," Transportmetrica A: Transport Science, vol. 15, no. 2, pp. 722-748, 2018.

[22] R. Guimera and L. A. N. Amaral, "Modeling the world-wide airport network," The European Physical Journal B-Condensed Matter, vol. 38, pp. 381-385, 2004.

[23] B. Jiang, Y. Duan, F. Lu, T. Yang, and J. Zhao, “Topological structure of urban street networks from the perspective of degree correlations," Environment and Planning B: Planning and Design, vol. 41, no. 5, pp. 813-828, 2014.

[24] A. Kirkley, H. Barbosa, M. Barthelemy, and G. Ghoshal, "From the betweenness centrality in street networks to structural invariants in random planar graphs," Nature Communications, vol. 9, p. 2051, 2018.

[25] A. P. Masucci, K. Stanilov, and M. Batty, "Exploring the evolution of London's street network in the information space: a dual approach," Physical Review E, vol. 89, no. 012805, pp. 1-7, 2014.

[26] X. Zhan and S. V. Ukkusuri, "Dynamics of functional failures and recovery in complex road networks," Physical Review E, vol. 96, no. 052301, pp. 1-16, 2017.

[27] Y. Duan and F. Lu, "Robustness of city road networks at different granularities," Physica A: Statistical Mechanics and Its Applications, vol. 411, pp. 21-34, 2014.

[28] M. Bellingeri, D. Bevacqua, F. Scotognella, Z.-M. Lu, and D. Cassi, "Efficacy of local attack strategies on the Beijing road complex weighted network," Physica A: Statistical Mechanics and Its Applications, vol. 510, pp. 316-328, 2018.

[29] C. Von Ferber, T. Holovatch, Y. Holovatch, and V. Palchykov, "Network harness: metropolis public transport," Physica A: Statistical Mechanics and Its Applications, vol. 380, pp. 585591, 2007.

[30] M. Rosvall, A. Trusina, P. Minnhagen, and K. Sneppen, "Networks and cities: an information perspective," Physical Review Letters, vol. 94, no. 2, 2005.

[31] M. Barthélemy, "Spatial networks," Physics Reports, vol. 499, no. 1-3, pp. 1-101, 2011.

[32] W.-L. Shang, Y. Chen, C. Song, and W. Y. Ochieng, "Robustness analysis of urban road networks from topological and operational perspectives," Mathematical Problems in Engineering, vol. 2020, Article ID 5875803, 12 pages, 2020.

[33] P. Crucitti, V. Latora, M. Marchiori, and A. Rapisarda, "Efficiency of scale-free networks: error and attack tolerance," Physica A: Statistical Mechanics and Its Applications, vol. 320, pp. 622-642, 2003.

[34] J. Wang, H. Mo, F. Wang, and F. Jin, "Exploring the network structure and nodal centrality of China's air transport network: a complex network approach," Journal of Transport Geography, vol. 19, no. 4, pp. 712-721, 2011.

[35] B. Berche, C. V. Ferber, T. Holovatch, and Y. Holovatch, "Transportation network stability: a case study of city transit," Advances in Complex Systems, vol. 15, no. supp01, Article ID 1250063, 2012.

[36] W. Li and X. Cai, "Statistical analysis of airport network of China," Physical Review E, vol. 69, 2004.

[37] V. Latora and M. Marchiori, "Is the Boston subway a smallworld network?" Physica A: Statistical Mechanics and Its Applications, vol. 314, no. 1-4, pp. 109-113, 2002.

[38] G. Sabidussi, "The centrality index of a graph," Psychometrika, vol. 31, no. 4, pp. 581-603, 1966.

[39] X. Sun, S. Wandelt, and F. Linke, “Topological properties of the air navigation route system using complex network theory," in Proceedings of the 6th International Conference on Research in Air Transportation (ICRAT2014), Istanbu Technical University, Istanbul, Turkey, May 2014.

[40] P. Crucitti, V. Latora, and M. Marchiori, "A topological analysis of the Italian electric power grid," Physica A: Statistical Mechanics and Its Applications, vol. 338, no. 1-2, pp. 92-97, 2004.

[41] R. Albert, H. Jeong, and A.-L. Barabási, "Error and attack tolerance of complex networks," Nature, vol. 406, no. 6794, pp. 378-382, 2000. 
[42] F. Karkubthy, "Chains, in everything is different," https://djjrcourses.wdfiles.com/local--files/soc180\%3Akarinthy-chainlinks/Karinthy-Chain-Links_1929.pdf, 1929.

[43] I. d. S. Pool and M. Kochen, Contacts and Influence, Social Network, Menlo park, CA, USA, 1978.

[44] S. Milgram, "The small world problem," Psychology Today, vol. 2, pp. 60-67, 1967.

[45] M. E. J. Newman, "The structure and function of complex networks," SIAM Review, vol. 45, no. 2, pp. 167-256, 2003.

[46] M. E. J. Newman, S. H. Strogatz, and D. J. Watts, "Random graphs with arbitrary degree distributions and their applications," Physical Review E, vol. 64, Article ID 026118, 2001.

[47] S. Boccaletti, V. Latora, Y. Moreno, M. Chavez, and D. Hwang, "Complex networks: structure and dynamics," Physics Reports, vol. 424, no. 4-5, pp. 175-308, 2006.

[48] R. Albert and A.-L. Barabási, "Statistical mechanics of complex networks," Reviews of Modern Physics, vol. 74, no. 1, pp. 47-97, 2002.

[49] R. Guimerà, M. Sales-Pardo, and L. A. N. Amaral, "A network-based method for target selection in metabolic networks," Bioinformatics, vol. 23, no. 13, pp. 1616-1622, 2007.

[50] A. Vázquez, R. Pastor-Satorras, and A. Vespignani, "Largescale topological and dynamical properties of the Internet," Physical Review E, vol. 65, no. 6, 2002.

[51] S. Fortunato, "Community detection in graphs," Physics Reports, vol. 486, no. 3-5, pp. 75-174, 2010.

[52] A. Lancichinetti, F. Radicchi, J. J. Ramasco, and S. Fortunato, "Finding statistically significant communities in networks," PLoS One, vol. 6, no. 4, Article ID e18961, 2011.

[53] M. E. J. Newman and T. P. Peixoto, "Generalized communities in networks," Physical Review Letters, vol. 115, pp. 1-5, 2015.

[54] B. Krishnamurthy and J. Wang, "On network-aware clustering of Web clients," ACM SIGCOMM Computer Communication Review, vol. 30, no. 4, pp. 97-110, 2000.

[55] K. P. Reddy, M. Kitsuregawa, P. Sreekanth, and S. Srinivasa Rao, "A graph based approach to extract a neighborhood customer community for collaborative filtering," in Proceedings of the Second International Workshop on Databases in Networked Information Systems, pp. 188-200, Springer-Verlag, Aizu, Japan, December 2002.

[56] F. Wu and B. A. Huberman, "Finding communities in linear time: a physics approach," The European Physical Journal B-Condensed Matter, vol. 38, no. 2, pp. 331-338, 2004.

[57] G. Petri, P. Expert, H. J. Jensen, and J. W. Polak, "Entangled communities and spatial synchronization lead to criticality in urban traffic," Scientific Reports, vol. 3, pp. 1-8, 2013.

[58] A. Lancichinetti, F. Radicchi, and J. J. Ramasco, "Statistical significance of communities in networks," Physical Review E, vol. 81, Article ID 046110, 2010.

[59] G. Casella and R. L. Berger, Statistical Inference, Duxbury Thomson Learning, Belmont, CA, USA, 2nd edition, 2001.

[60] P. Holme, B. J. Kim, C. N. Yoon, and S. K. Han, "Attack vulnerability of complex networks," Physical Review E, vol. 65, no. 5, 2002.

[61] P. Crucitti, V. Latora, M. Marchiori, and A. Rapisarda, "Error and attack tolerance of complex networks," Physica A: Statistical Mechanics and Its Applications, vol. 340, no. 1-3, pp. 388-394, 2004. 